\title{
One-shot lossy quantum data compression
}

\author{
Nilanjana Datta \\ Statistical Laboratory, University of Cambridge, \\ Cambridge CB3 OWB, United Kingdom \\ Joseph M. Renes and Renato Renner \\ Institute for Theoretical Physics, ETH Zurich, \\ 8093 Zürich, Switzerland \\ Mark M. Wilde \\ School of Computer Science, McGill University, \\ Montreal, Quebec H3A 2A7, Canada
}

July 16, 2018

\begin{abstract}
We provide a framework for one-shot quantum rate distortion coding, in which the goal is to determine the minimum number of qubits required to compress quantum information as a function of the probability that the distortion incurred upon decompression exceeds some specified level. We obtain a one-shot characterization of the minimum qubit compression size for an entanglement-assisted quantum rate-distortion code in terms of the smooth max-information, a quantity previously employed in the one-shot quantum reverse Shannon theorem. Next, we show how this characterization converges to the known expression for the entanglement-assisted quantum rate distortion function for asymptotically many copies of a memoryless quantum information source. Finally, we give a tight, finite blocklength characterization for the entanglementassisted minimum qubit compression size of a memoryless isotropic qubit source subject to an average symbol-wise distortion constraint.
\end{abstract}

\section{Introduction}

The reliable compression of data is essential for the efficient use of available storage or communication resources. In one of the first breakthroughs of quantum information theory, Schumacher [30] proved that the von Neumann entropy of a memoryless quantum information source is the optimal rate at which we can compress it. This data compression limit was evaluated under the requirement that the compression-decompression scheme is asymptotically lossless, in the sense that the information emitted by the source is recovered with arbitrarily good accuracy in the limit of asymptotically many copies of the source.

However, one could envisage scenarios in which some imperfection in the recovered information would be tolerable or even necessary. The characterization of the trade-off between an allowed distortion and the compression rate is the subject of quantum rate distortion theory. Its classical counterpart was developed by Shannon [31, and the trade-off is given by a rate-distortion function, which is defined as the minimum rate of compression for a given distortion, with respect to a 
suitably defined distortion measure. To our knowledge, there are at least two important reasons for developing the theory of lossy quantum data compression:

1. One might need to compress a quantum information source at a rate smaller than its von Neumann entropy. This is necessary, for example, in the case where there is insufficient storage available, or if one needs to transmit information emitted by a source over a channel whose quantum capacity is smaller than the von Neumann entropy of the source. The strong converse to Schumacher's theorem implies that there is no trade-off possible between the rate of compression and the error incurred in recovery in the asymptotic limit (see Theorem I.19 of [42]). That is, there cannot be a "rate-error" trade-off because if one compresses at a rate below the von Neumann entropy, then the fidelity between the initial and recovered state approaches zero exponentially in the number of copies of the source. In spite of this "nogo" theorem, the theory of quantum rate distortion shows that there can be a fundamental trade-off between rate and distortion for a suitably defined distortion measure.

2. Allowing a finite distortion in the recovered data is essential for some continuous-variable quantum information sources (see [39] and references therein) for which the requirement of arbitrarily good accuracy becomes meaningless 1 That is, we would like to have a theory that characterizes the compression of analog quantum information into digital quantum information along with the distortion incurred in doing so.

The first paper to discuss rate distortion in the quantum realm was by Barnum [3]. He introduced a definition of the quantum rate-distortion function as the lowest rate at which a sender can compress a memoryless quantum source under some distortion constraint. The main result of his paper is a lower bound on the quantum rate distortion function in terms of a well-known entropic quantity, namely, the coherent information. Even though Barnum's result was the first in quantum rate distortion theory, it is unsatisfactory since the bound is obviously loose - the coherent information can be negative, whereas the quantum rate distortion function is defined operationally to be non-negative. Tighter, non-negative lower bounds were found in later work, by allowing for assisting resources such as entanglement assistance [15] or a side classical channel [40].

Even though classical rate distortion theory has been an area of active research, its quantum analogue had received very little attention, there being only a few results on it since Barnum's work [18, 9, 26]. In the past few years, however, there has been a revival of interest in quantum rate distortion theory, and quite a few new results have been obtained [15, 16, 40]. These later works found various expressions for quantum rate distortion functions, both in the absence and presence of auxiliary resources, which can be exploited in the data compression task.

In all prior work on quantum rate distortion theory, the rate-distortion functions were evaluated in the limit of asymptotically many copies of a memoryless quantum information source. Since the data compression rates in those works were achieved using block codes, this corresponds to the limit $n \rightarrow \infty$, where $n$ denotes the length of the block code. These results then give useful bounds in an idealized setting, but they are not particularly helpful in characterizing the rate-distortion trade-off for more realistic settings, such as the finite blocklength setting or one in which the source is not memoryless.

\footnotetext{
${ }^{1}$ An important exception here is the case of a bosonic thermal source, which has a discrete representation in the orthonormal photon-number basis. Thus, Schumacher compression of a bosonic thermal source is indeed possible, even though its representation in the coherent-state basis is continuous.
} 
A more fundamental problem, of both theoretical and practical interest, is to find bounds on rate distortion functions for a given distortion $D \geq 0$ and an "excess-distortion" probability $\varepsilon>0$. For example, consider the classical case. Let a source be described by a random variable $X$ taking values in a finite alphabet $\mathcal{X}$. We would like to find the minimum number of bits to which we can compress this source, such that the probability of exceeding a distortion level $D$ is no larger than some small $\varepsilon>0$ :

$$
\operatorname{Pr}\{d(X,(\mathcal{D} \circ \mathcal{E})(X))>D\}:=\sum_{x \in \mathcal{X}} p_{X}(x) I\{d(x,(\mathcal{D} \circ \mathcal{E})(x))>D\} \leq \varepsilon,
$$

where $I\{\cdot\}$ denotes the indicator function, $d(\cdot, \cdot)$ is a distortion measure, and $\mathcal{E}$ and $\mathcal{D}$ are the respective encoder and decoder for the scheme. We could then evaluate such a bound for a source that is invoked a finite number of times. In the classical case, in certain applications, relatively short blocklengths are in fact common, both due to delay and complexity constraints, and we would expect similar constraints to apply in the quantum case. In this vein, Kostina and Verdú recently obtained bounds on the minimum achievable rate of classical data compression as a function of blocklength $n$ and excess distortion probability $\varepsilon$ [25].

\section{Overview of Results}

In this paper, we contribute the following results:

- We first establish a framework for one-shot quantum rate-distortion theory. This includes some basic definitions and the notion of an excess-distortion projector, which is derived from a distortion observable 2 The definitions apply in settings where either there is no assisting resource or entanglement assistance is available.

- We obtain two lower (converse) bounds (Propositions 7 and 9) on the minimum qubit compression size, which is the minimum number of qubits needed to compress the source state such that a receiver can recover it up to some specified excess-distortion probability. The bounds apply in the entanglement-assisted setting, and as such, they apply in the unassisted case as well. These bounds are given in terms of quantities defined in the smooth-entropy framework of one-shot information theory (see [29, 33, 19, 14, and references therein) and are proved by employing ideas from quantum hypothesis testing (see, e.g., [19] and references therein). One of our converse bounds (Proposition 9) can in fact be viewed as a generalization of a converse bound proved in the classical case by Kostina and Verdú [25].

- Achievability bounds in Sections 6.1 and 6.2 are proved using a one-shot version of the quantum reverse Shannon (channel simulation) theorem [7]. A channel simulation theorem provides bounds on the minimum number of qubits that a sender (say, Alice) needs to send to a receiver (say, Bob) in order to simulate a quantum channel up to a finite accuracy. A channel simulation strategy then leads to bounds on the one-shot entanglement-assisted quantum rate-distortion function by choosing the simulated channel to depend on the distortion measure.

\footnotetext{
${ }^{2} \mathrm{~A}$ distortion observable is a generalization of the distortion measure used in classical rate-distortion theory.
} 
- Theorem[11]unifies the above results, demonstrating that the smooth max-information from [7] provides a characterization of the one-shot entanglement-assisted rate distortion function up to logarithmic correction terms.

- The bounds obtained in the one-shot setting readily yield bounds on the minimum qubit compression rate for finite blocklength, for a memoryless quantum information source. In the limit of asymptotically large blocklength $(n \rightarrow \infty)$, these bounds converge independently to the known single-letter expression for the entanglement-assisted quantum rate distortion function [15], given in terms of the quantum mutual information.

- We demonstrate how a good channel simulation protocol, in which the simulated channel depends on the distortion measure, leads to a rate distortion protocol that performs well with respect to the excess-distortion probability criterion (see Lemma 19 for details).

- Our final contributions in Section 10 are 1) to evaluate one of the aforementioned converse bounds for the special case of an isotropic qubit source and an entanglement-fidelity based distortion measure and 2) to outline a quantum teleportation strategy that nearly meets this converse bound in the finite blocklength regime. Even though this latter strategy is rather simple, it represents the first example in quantum rate distortion theory where a strategy other than channel simulation is used to achieve non-trivial compression rates.

This paper is organized as follows. In the next section, we introduce necessary notation and definitions, especially for the entropic quantities arising in the statements of the theorems. The rest of the paper proceeds in the order of the results mentioned above, and then we end with a conclusion that summarizes our results and points to open questions for future research.

\section{$3 \quad$ Notation and Definitions}

Let $\mathcal{B}(\mathcal{H})$ denote the algebra of linear operators acting on a finite-dimensional Hilbert space $\mathcal{H}$, let $\mathcal{B}(\mathcal{H})_{+}$denote the set of positive semi-definite operators on $\mathcal{H}$, and let $\mathcal{D}(\mathcal{H}) \subset \mathcal{B}(\mathcal{H})_{+}$denote the set of density operators (or states), i.e., positive semi-definite operators of unit trace. Furthermore, we define the set of subnormalized states $\mathcal{D}_{\leq}(\mathcal{H}):=\left\{\rho \in \mathcal{B}(\mathcal{H})_{+}: \operatorname{Tr} \rho \leq 1\right\}$. Throughout this paper, for simplicity, we restrict our considerations to finite-dimensional Hilbert spaces, and we denote the dimension of a Hilbert space $\mathcal{H}_{A}$ as $|A|$. 3

For states $\rho, \sigma \in \mathcal{D}(\mathcal{H})$, the quantum fidelity is defined as

$$
F(\rho, \sigma):=\|\sqrt{\rho} \sqrt{\sigma}\|_{1},
$$

where $\|A\|_{1}=\operatorname{Tr}\left(\sqrt{A A^{\dagger}}\right)$. Uhlmann characterized the fidelity as the maximal overlap between any two purifications $\left|\phi_{\rho}\right\rangle$ and $\left|\phi_{\sigma}\right\rangle$ of $\rho$ and $\sigma$, respectively [36]:

$$
F(\rho, \sigma)=\max _{\left|\phi_{\rho}\right\rangle,\left|\phi_{\sigma}\right\rangle}\left|\left\langle\phi_{\rho} \mid \phi_{\sigma}\right\rangle\right| \text {. }
$$

Thus, the square of the fidelity has an operational interpretation as the optimal probability with which a purification of $\rho$ would pass a test for being a purification of $\sigma$ [24. Since all purifications are

\footnotetext{
${ }^{3}$ However, note that none of our bounds depend on the dimension of the input space, so that our results may easily be generalized to cases where the data to be compressed is infinite-dimensional. We leave this consideration for future work, where one should be able to use the methods from [21].
} 
related by an isometric operation on the purifying system, Uhlmann's characterization is equivalent to the following one:

$$
F(\rho, \sigma)=\max _{U}\left|\left\langle\phi_{\rho}\left|\left(U \otimes I_{\mathcal{H}}\right)\right| \phi_{\sigma}\right\rangle\right|,
$$

where $\left|\phi_{\rho}\right\rangle$ and $\left|\phi_{\sigma}\right\rangle$ are now two fixed purifications of $\rho$ and $\sigma$, respectively, and the optimization is over all isometries acting on the purifying system. The fact that (2) is equal to (3) is known as Uhlmann's theorem. The trace distance between two states $\rho$ and $\sigma$ is defined as follows: $\|\rho-\sigma\|_{1}$, and the fidelity and trace distance are related by the Fuchs-van-de-Graaf inequalities [20]:

$$
1-F(\rho, \sigma) \leq \frac{1}{2}\|\rho-\sigma\|_{1} \leq \sqrt{1-(F(\rho, \sigma))^{2}} .
$$

Moreover, for $\rho, \sigma \in \mathcal{D}_{\leq}(\mathcal{H})$ let $\bar{F}(\rho, \sigma)$ denote the generalized fidelity [35]:

$$
\bar{F}(\rho, \sigma)=F(\rho, \sigma)+\sqrt{(1-\operatorname{Tr} \rho)(1-\operatorname{Tr} \sigma)} .
$$

Observe that the generalized fidelity reduces to the standard fidelity in (2) if at least one of the two states is normalized. The purified distance quantifies the distance between any two subnormalized states $\rho, \sigma \in \mathcal{D}_{\leq}(\mathcal{H})[35]$ :

$$
P(\rho, \sigma):=\sqrt{1-(\bar{F}(\rho, \sigma))^{2}} .
$$

We denote a quantum channel, i.e., a completely positive trace-preserving (CPTP) map $\mathcal{E}$ : $\mathcal{B}\left(\mathcal{H}_{A}\right) \mapsto \mathcal{B}\left(\mathcal{H}_{B}\right)$ simply as $\mathcal{E}_{A \rightarrow B}$. Similarly, we denote an isometry $U: \mathcal{H}_{A} \mapsto \mathcal{H}_{B} \otimes \mathcal{H}_{C}$ simply as $U_{A \rightarrow B C}$.

The von Neumann entropy of a state $\rho \in \mathcal{D}\left(\mathcal{H}_{A}\right)$ is given by $H(A)_{\rho}:=-\operatorname{Tr}(\rho \log \rho)$. Throughout this paper we take the logarithm to base 2 . For a bipartite state $\rho_{A B} \in \mathcal{D}\left(\mathcal{H}_{A B}\right)$, the conditional entropy of $A$ given $B$, and the quantum mutual information between $A$ and $B$ are respectively given by:

$$
\begin{aligned}
H(A \mid B)_{\rho} & :=H(A B)_{\rho}-H(B)_{\rho}, \\
I(A ; B)_{\rho} & :=H(A)_{\rho}+H(B)_{\rho}-H(A B)_{\rho},
\end{aligned}
$$

where $H(A)_{\rho}$ denotes the von Neumann entropy of the reduced state $\rho_{A}=\operatorname{Tr}_{B}\left(\rho_{A B}\right)$. Furthermore, for $\rho \in \mathcal{D}(\mathcal{H})$ and $\sigma \in \mathcal{B}_{+}(\mathcal{H})$, such that $\operatorname{supp} \rho \subseteq \operatorname{supp} \sigma$, the quantum relative entropy is defined as

$$
D(\rho \| \sigma)=\operatorname{Tr}(\rho \log \rho)-\operatorname{Tr}(\rho \log \sigma) .
$$

We also make use of several other entropic quantities having their origin in the work of Renner [29]. The max-relative entropy of a subnormalized state $\rho \in \mathcal{D}_{\leq}(\mathcal{H})$ and an operator $\sigma \in \mathcal{B}(\mathcal{H})_{+}$ is defined as [13]

$$
D_{\max }(\rho \| \sigma):=\min \left\{\gamma: \rho \leq 2^{\gamma} \sigma\right\} .
$$

For any $\varepsilon>0$, the smooth max-relative entropy is given by

$$
D_{\max }^{\varepsilon}(\rho \| \sigma):=\min _{\bar{\rho} \in \mathcal{B}^{\varepsilon}(\rho)} D_{\max }(\bar{\rho} \| \sigma),
$$

where $\mathcal{B}^{\varepsilon}(\rho)$ denotes a ball of subnormalized states around $\rho \in \mathcal{D}_{\leq}(\mathcal{H})$ :

$$
\mathcal{B}^{\varepsilon}(\rho):=\left\{\bar{\rho} \in \mathcal{D}_{\leq}(\mathcal{H}): P(\rho, \bar{\rho}) \leq \varepsilon\right\}
$$


The conditional min-entropy of $A$ given $B$ for $\rho_{A B} \in \mathcal{D}_{\leq}\left(\mathcal{H}_{A B}\right)$ is defined as

$$
H_{\min }(A \mid B)_{\rho}:=-\min _{\sigma_{B} \in \mathcal{D}\left(\mathcal{H}_{B}\right)} D_{\max }\left(\rho_{A B} \| I_{A} \otimes \sigma_{B}\right)
$$

If the system $B$ is trivial, then this reduces to $H_{\min }(A)_{\rho}=-\log \left\|\rho_{A}\right\|_{\infty}$, where $\|\cdot\|_{\infty}$ denotes the operator norm. The max-information that $B$ has about $A$ for $\rho_{A B} \in \mathcal{D}_{\leq}\left(\mathcal{H}_{A B}\right)$ is defined as [7]

$$
I_{\max }(A ; B)_{\rho}=\min _{\sigma_{B} \in \mathcal{D}\left(\mathcal{H}_{B}\right)} D_{\max }\left(\rho_{A B} \| \rho_{A} \otimes \sigma_{B}\right)
$$

For any $\varepsilon \geq 0$, the smooth versions of the above quantities are defined as follows:

$$
\begin{aligned}
H_{\min }^{\varepsilon}(A \mid B)_{\rho} & :=\max _{\bar{\rho}_{A B} \in \mathcal{B}^{\varepsilon}\left(\rho_{A B}\right)} H_{\min }(A \mid B)_{\bar{\rho}}, \\
I_{\max }^{\varepsilon}(A ; B)_{\rho} & :=\min _{\bar{\rho}_{A B} \in \mathcal{B}^{\varepsilon}\left(\rho_{A B}\right)} I_{\max }(A ; B)_{\bar{\rho}} .
\end{aligned}
$$

For sequences of tensor power states, the (conditional) von Neumann entropy and the quantum mutual information are equal to the smooth entropy quantities defined above in an asymptotic limit [34, 33]. That is, for a sequence of states $\left\{\rho_{A^{n} B^{n}}\right\}_{n \geq 1}$, where $\rho_{A^{n} B^{n}}=\rho_{A B}^{\otimes n} \in \mathcal{D}\left(\mathcal{H}_{A B}^{\otimes n}\right)$, it is known that, for $\varepsilon \in(0,1)$,

$$
\begin{aligned}
& \lim _{n \rightarrow \infty} \frac{1}{n} H_{\min }^{\varepsilon}\left(A^{n} \mid B^{n}\right)_{\rho}=H(A \mid B)_{\rho}, \\
& \lim _{n \rightarrow \infty} \frac{1}{n} I_{\max }^{\varepsilon}\left(A^{n} ; B^{n}\right)_{\rho}=I(A ; B)_{\rho} .
\end{aligned}
$$

Furthermore, for any $\varepsilon \geq 0$ and $\rho \in \mathcal{D}\left(\mathcal{H}_{A}\right)$, we define

$$
H_{0}^{\varepsilon}(A)_{\rho}:=\min _{\bar{\rho} \in \mathcal{B}^{\varepsilon}(\rho)} H_{0}(A)_{\bar{\rho}}
$$

where $H_{0}(A)_{\bar{\rho}}=\log \operatorname{rank} \bar{\rho}$ denotes the Rényi entropy of order zero. It is also known that for a sequence of states $\left\{\rho_{A^{n}}\right\}_{n \geq 1}$, with $\rho_{A^{n}}=\rho_{A}^{\otimes n}$, and for every $\varepsilon \in(0,1)$

$$
\lim _{n \rightarrow \infty} \frac{1}{n} H_{0}^{\varepsilon}\left(A^{n}\right)_{\rho}=H(A)_{\rho} .
$$

(The above result is in [42]. It also follows from (4.2) and Result 6 of [33].)

We shall also make use of the hypothesis testing relative entropy. First, let $\beta_{\varepsilon}(\rho \| \sigma)$ denote the optimal type II error probability in a quantum hypothesis test that distinguishes between $\rho$ and some other state $\sigma$, when the type I error probability is fixed to be less than $\varepsilon$ :

$$
\beta_{\varepsilon}(\rho \| \sigma):=\min _{\Lambda}\{\operatorname{Tr}\{\Lambda \sigma\}: 0 \leq \Lambda \leq I, \operatorname{Tr}\{\Lambda \rho\} \geq 1-\varepsilon\} .
$$

Wang and Renner 38] define the hypothesis testing relative entropy as

$$
D_{H}^{\varepsilon}(\rho \| \sigma)=-\log \beta_{\varepsilon}(\rho \| \sigma) .
$$

Various properties of this quantity were explored in [19], one of which is the following useful lemma: 
Lemma 1 Let $\rho \in \mathcal{D}(\mathcal{H}), \sigma \in \mathcal{B}_{+}(\mathcal{H})$ and $0<\varepsilon \leq 1$. Then

$$
D_{\max }^{\sqrt{2(1-\varepsilon)}}(\rho \| \sigma)+\log \left(\frac{1}{1-\varepsilon}\right) \leq D_{H}^{\varepsilon}(\rho \| \sigma) \leq D_{\max }(\rho \| \sigma)+\log \left(\frac{1}{1-\varepsilon}\right) .
$$

We also make use of the gentle operator lemma [41, 28] and another lemma that follows from a variational characterization of the trace distance:

Lemma 2 (Gentle Operator) Consider a density operator $\rho$ and a measurement operator $\Lambda$ satisfying $0 \leq \Lambda \leq I$. Suppose that the measurement operator $\Lambda$ has a high probability of detecting the state $\rho$ :

$$
\operatorname{Tr}\{\Lambda \rho\} \geq 1-\varepsilon,
$$

where $1 \geq \varepsilon>0$ (the probability is high only if $\varepsilon$ is close to zero). Then the subnormalized state $\sqrt{\Lambda} \rho \sqrt{\Lambda}$ is $2 \sqrt{\varepsilon}$-close to the original state $\rho$ in trace distance:

$$
\|\rho-\sqrt{\Lambda} \rho \sqrt{\Lambda}\|_{1} \leq 2 \sqrt{\varepsilon}
$$

Lemma 3 Suppose we have two quantum states $\rho$ and $\sigma$ and an operator $\Lambda$ where $0 \leq \Lambda \leq I$. Then

$$
\operatorname{Tr}\{\Lambda \rho\} \geq \operatorname{Tr}\{\Lambda \sigma\}-\frac{1}{2}\|\rho-\sigma\|_{1}
$$

\section{One-Shot Quantum Rate Distortion Coding}

In this section, we establish definitions for the most general "one-shot" setting of quantum rate distortion coding, in which there are no assumptions about the structure of the source state or the distortion observable (defined below). Throughout this paper, we work in a communication paradigm, in which a sender Alice has access to a quantum information source, and the goal is for her to use as few noiseless qubit channels as possible to transmit a compressed version of the source so that a receiver Bob can recover it up to some distortion. This section also establishes definitions for the entanglement-assisted setting, in which Alice and Bob share entanglement and can exploit this resource in their compression-decompression task.

\subsection{Unassisted One-Shot Quantum Rate-Distortion Code}

A quantum source is described by some density operator $\rho \in \mathcal{D}\left(\mathcal{H}_{A}\right)$. A lossy quantum data compression code consists of an encoding map $\mathcal{E}: \mathcal{B}\left(\mathcal{H}_{A}\right) \rightarrow \mathcal{B}\left(\mathcal{H}_{M}\right)$, which we denote as $\mathcal{E}_{A \rightarrow M}$ for short, where $\mathcal{H}_{M}$ is a compressed space spanned by an orthonormal basis $\{|1\rangle, \ldots,|M\rangle\}$. The decoding map is defined as $\mathcal{D}: \mathcal{B}\left(\mathcal{H}_{M}\right) \rightarrow \mathcal{B}\left(\mathcal{H}_{B}\right)$ and denoted by $\mathcal{D}_{M \rightarrow A}$. Let $\mathcal{H}_{R}$ be a purifying Hilbert space, so that $\left|\varphi^{\rho}\right\rangle_{R A} \in \mathcal{H}_{R} \otimes \mathcal{H}_{A}$ is a purification of the source state $\rho$. The joint state of the reference and the output after the action of the encoding and decoding maps is

$$
\left(\operatorname{id}_{R} \otimes\left(\mathcal{D}_{M \rightarrow B} \circ \mathcal{E}_{A \rightarrow M}\right)\right)\left(\varphi_{R A}^{\rho}\right) .
$$

A distortion observable $\Delta_{R B}$ is some operator in $\mathcal{B}_{+}\left(\mathcal{H}_{R} \otimes \mathcal{H}_{B}\right)$ that quantifies the performance of a lossy quantum compression code [44, 9, 16, 40. Since $\Delta_{R B}$ is positive semi-definite, it has a spectral decomposition of the following form:

$$
\Delta_{R B}=\sum_{z} d_{z}\left|\phi_{z}\right\rangle\left\langle\left.\phi_{z}\right|_{R B}\right.
$$


where $d_{z} \geq 0$ for all $z$. In this paper, we assume a finite bound on the maximum eigenvalue of the distortion observable $\Delta_{R B}$ :

$$
d_{\max }:=\left\|\Delta_{R B}\right\|_{\infty}<\infty .
$$

In order for a distortion observable to quantify the deviation of a protocol's output state from the source state, it should depend on the source state in some way.

Let $\left(\Pi_{>D}\right)_{R B}$ denote the excess-distortion projector associated to $\Delta_{R B}$. It is equal to the projection onto a subspace of $\mathcal{H}_{R} \otimes \mathcal{H}_{B}$ spanned by eigenvectors of $\Delta_{R B}$ whose corresponding eigenvalues are larger than some non-negative number $D$ :

$$
\left(\Pi_{>D}\right)_{R B}:=\sum_{z: d_{z}>D}\left|\phi_{z}\right\rangle\left\langle\left.\phi_{z}\right|_{R B} .\right.
$$

The excess-distortion projector generalizes the indicator function used to define the excess-distortion probability in the classical case (where the indicator function selects the event in which the distortion exceeds $D$, as in (10) $[22,27,25]$.

We have the following definition of a quantum rate-distortion code with performance measured by the excess-distortion probability:

Definition 4 An $(M, D, \varepsilon)$ quantum rate distortion code for $\left\{\mathcal{H}_{A}, \mathcal{H}_{B}, \rho, \Delta_{R B}\right\}$ is a code with $\left|\mathcal{H}_{M}\right|=M$ and such that

$$
\operatorname{Tr}\left\{\left(\Pi_{>D}\right)_{R B}\left(\operatorname{id}_{R} \otimes\left(\mathcal{D}_{M \rightarrow B} \circ \mathcal{E}_{A \rightarrow M}\right)\right)\left(\varphi_{R A}^{\rho}\right)\right\} \leq \varepsilon .
$$

The minimum achievable code size at excess-distortion probability $\varepsilon$ and distortion $D$ is defined by

$$
M^{*}\left(\rho, \Delta_{R B}, D, \varepsilon\right):=\min \left\{M: \exists \text { an }(M, D, \varepsilon) \text { code for }\left\{\mathcal{H}_{A}, \mathcal{H}_{B}, \rho, \Delta_{R B}\right\}\right\} .
$$

We refer to the quantity $\log \left(M^{*}\left(\rho, \Delta_{R B}, D, \varepsilon\right)\right)$ as the minimum qubit compression size 4

The minimum achievable code size is a quantity that is difficult to compute, and one of the goals of the present paper is to provide useful bounds on it.

The special case $D=0$ and $\Delta_{R B}=I_{R B}-|\varphi\rangle\left\langle\left.\varphi\right|_{R B}\right.$ corresponds to almost lossless quantum data compression (one-shot Schumacher compression). Indeed, such a choice leads to the condition in (27) becoming

$$
\left\langle\left.\varphi\right|_{R B}\left(\operatorname{id}_{R} \otimes\left(\mathcal{D}_{M \rightarrow B} \circ \mathcal{E}_{A \rightarrow M}\right)\right)\left(\varphi_{R A}^{\rho}\right) \mid \varphi\right\rangle_{R B} \geq 1-\varepsilon,
$$

which is the usual entanglement-fidelity based criterion employed in Schumacher compression [30].

Definition 4 captures the critical idea behind formulating a good one-shot framework for quantum rate distortion: the output of the protocol is allowed to deviate beyond a distortion specified by $D$, but only with a probability less than $\varepsilon$.

We could also use a mean distortion criterion, which corresponds to the more traditional formulation in prior work on quantum rate distortion coding [3, 18, 15]. For a given distortion observable $\Delta_{R B}$, the mean distortion of the source state under a CPTP map $\mathcal{N}_{A \rightarrow B}$ is defined as follows:

$$
\delta_{\text {mean }}\left(\rho, \mathcal{N}_{A \rightarrow B}, \Delta_{R B}\right):=\operatorname{Tr}\left(\Delta_{R B} \omega_{R B}\right),
$$

where

$$
\omega_{R B}:=\left(\operatorname{id}_{R} \otimes \mathcal{N}_{A \rightarrow B}\right) \varphi_{R A}^{\rho}
$$

\footnotetext{
${ }^{4}$ The minimum qubit compression size should really be defined as $\left\lceil\log \left(M^{*}\left(\rho, \Delta_{R B}, D, \varepsilon\right)\right)\right\rceil$, but we will omit the inclusion of the "ceiling" in the rest of the paper for simplicity.
} 
Definition 5 An $\langle M, D\rangle$ quantum rate distortion code for $\left\{\mathcal{H}_{A}, \mathcal{H}_{B}, \rho, \Delta_{R B}\right\}$ is a code with $\left|\mathcal{H}_{M}\right|=$ $M$ and mean distortion

$$
\delta_{\text {mean }}\left(\rho, \mathcal{D}_{M \rightarrow B} \circ \mathcal{E}_{A \rightarrow M}, \Delta_{R B}\right) \leq D .
$$

The minimum achievable code size at mean distortion $D$ is defined by

$$
M^{*}\left(\rho, \Delta_{R B}, D\right):=\min \left\{M: \exists \text { an }\langle M, D\rangle \text { code for }\left\{\mathcal{H}_{A}, \mathcal{H}_{B}, \rho, \Delta_{R B}\right\}\right\}
$$

The minimum qubit compression size is equal to $\log \left(M^{*}\left(\rho, \Delta_{R B}, D\right)\right)$.

The excess-distortion probability is a stronger criterion for quantum rate distortion coding in the sense of the following lemmat5

Lemma 6 Suppose that there exists an $(M, D, \varepsilon)$ quantum rate distortion code for $\left\{\mathcal{H}_{A}, \mathcal{H}_{B}, \rho, \Delta_{R B}\right\}$. Then this code is also an $\left\langle M, D+d_{\max } \varepsilon\right\rangle$ quantum rate distortion code for $\left\{\mathcal{H}_{A}, \mathcal{H}_{B}, \rho, \Delta_{R B}\right\}$.

Proof. The proof of this statement easily follows by exploiting the following operator inequality:

$$
\begin{aligned}
\Delta_{R B} & =\sum_{z} d_{z}\left|\phi_{z}\right\rangle\left\langle\left.\phi_{z}\right|_{R B}\right. \\
& =\sum_{z: d_{z} \leq D} d_{z}\left|\phi_{z}\right\rangle\left\langle\left.\phi_{z}\right|_{R B}+\sum_{z: d_{z}>D} d_{z} \mid \phi_{z}\right\rangle\left\langle\left.\phi_{z}\right|_{R B}\right. \\
& \leq D I_{R B}+d_{\max }\left(\Pi_{>D}\right)_{R B} .
\end{aligned}
$$

This then leads to the statement of the lemma:

$$
\begin{aligned}
& \operatorname{Tr}\left\{\Delta_{R B}\left(\operatorname{id}_{R} \otimes\left(\mathcal{D}_{M \rightarrow B} \circ \mathcal{E}_{A \rightarrow M}\right)\right)\left(\varphi_{R A}^{\rho}\right)\right\} \\
& \leq D+d_{\max } \operatorname{Tr}\left\{\left(\Pi_{>D}\right)_{R B}\left(\operatorname{id}_{R} \otimes\left(\mathcal{D}_{M \rightarrow B} \circ \mathcal{E}_{A \rightarrow M}\right)\right)\left(\varphi_{R A}^{\rho}\right)\right\} \\
& \leq D+d_{\max } \varepsilon
\end{aligned}
$$

Thus, the excess-distortion probability is an alternative performance criterion related to the traditional mean distortion criterion, but more importantly, it leads to a meaningful one-shot extension of the traditional framework.

\subsection{Entanglement-Assisted One-Shot Quantum Rate-Distortion Code}

An entanglement-assisted quantum rate distortion code is defined similarly to an unassisted one, but the sender (Alice) and receiver (Bob) are allowed to share entanglement before the protocol begins [15]. Let $\Psi_{T_{A} T_{B}}$ denote the entangled state that they share, where Alice possesses system $T_{A}$ and Bob possesses system $T_{B}$, and note that the state can be an arbitrary entangled state. The protocol begins with Alice and Bob combining their systems $T_{A}$ and $T_{B}$ with the source state $\varphi_{R A}^{\rho}$, to produce

$$
\varphi_{R A}^{\rho} \otimes \Psi_{T_{A} T_{B}}
$$

\footnotetext{
${ }^{5}$ Note, however, that the excess-distortion probability and mean distortion criteria become essentially equivalent in the independent and identically distributed (i.i.d.) setting. This follows from Lemma 6 and Lemma 19.
} 
(This is a trivial "appending" CPTP map.) Alice then acts with an encoding map $\mathcal{E}_{A T_{A} \rightarrow M}$, and Bob acts with a decoding map $\mathcal{D}_{M T_{B} \rightarrow B}$, resulting in the state

$$
\left(\operatorname{id}_{R} \otimes\left(\mathcal{D}_{M T_{B} \rightarrow B} \circ \mathcal{E}_{A T_{A} \rightarrow M}\right)\right)\left(\varphi_{R A}^{\rho} \otimes \Psi_{T_{A} T_{B}}\right) .
$$

We can write the combined action of appending, encoding, and decoding as some CPTP map $\mathcal{N}_{A \rightarrow B}^{e a}$ :

$$
\mathcal{N}_{A \rightarrow B}^{e a}\left(\sigma_{A}\right):=\left(\mathcal{D}_{M T_{B} \rightarrow B} \circ \mathcal{E}_{A T_{A} \rightarrow M}\right)\left(\sigma_{A} \otimes \Psi_{T_{A} T_{B}}\right),
$$

for any input density operator $\sigma_{A}$. An $(M, D, \varepsilon)$ entanglement-assisted quantum rate distortion code and an $\langle M, D\rangle$ entanglement-assisted quantum rate distortion code are then defined analogously as in Definitions 4 and 5, respectively, with respect to the state in (29). The minimum achievable code sizes and minimum qubit compression sizes are defined analogously as well.

\section{Converse Bounds for One-Shot Entanglement-Assisted Quan- tum Rate Distortion Codes}

This section provides two general converse bounds that apply to one-shot entanglement-assisted quantum rate distortion codes. The first converse provides a bound in terms of $D_{H}^{1-\varepsilon^{\prime}}$ (where $\varepsilon^{\prime}$ is related to the excess-distortion probability) and thus is related to $D_{\max }^{\sqrt{2 \varepsilon^{\prime}}}$ by Lemma 1, We show in Section 9.2 that in the i.i.d. limit, the expression in this first converse is bounded from below by the known quantity for the entanglement-assisted quantum rate distortion (EA-QRD) function from [15].

The second converse in this section provides a bound in terms of $D_{H}^{\varepsilon}$ and can be seen as a direct quantum generalization of the Kostina-Verdú bound from [25]. We apply this bound in Section 10.1 to give a tight finite-blocklength characterization of the i.i.d. entanglement-assisted quantum rate distortion function for an isotropic qubit source. Although this second converse gives a tight characterization for this example, it is unclear to us if this converse generally converges in the i.i.d. limit to the known quantity from [15] for all quantum information sources.

Of course, since these converses provide lower bounds on the minimum qubit compression sizes of entanglement-assisted quantum rate distortion codes, they provide lower bounds for unassisted codes as well.

\subsection{A One-Shot Converse Bound}

Proposition 7 Let $\rho$ be the density operator characterizing a quantum information source, and let $\left|\varphi^{\rho}\right\rangle_{R A}$ be a purification of it. For any $(M, D, \varepsilon)$ entanglement-assisted quantum rate distortion code for $\left\{\mathcal{H}_{A}, \mathcal{H}_{B}, \rho, \Delta_{R B}\right\}$, we have the following lower bound on its minimum qubit compression size:

$$
\begin{aligned}
& \log M^{*}\left(\rho, \Delta_{R B}, D, \varepsilon\right) \\
& \geq \frac{1}{2} \min _{\mathcal{N}_{A \rightarrow B}} \max _{\sigma_{R A}} \min _{\psi_{B}}\left[D_{H}^{1-\varepsilon^{\prime}}\left(\left(\operatorname{id}_{R} \otimes \mathcal{N}_{A \rightarrow B}\right)\left(\varphi_{R A}^{\rho}\right) \| \sigma_{R} \otimes \psi_{B}\right)-D_{H}^{1-\varepsilon^{\prime \prime}}\left(\varphi_{R A}^{\rho} \| \sigma_{R A}\right)\right],
\end{aligned}
$$

where $\varepsilon^{\prime} \geq 2 \varepsilon, \varepsilon^{\prime \prime}:=\varepsilon^{\prime}\left(\frac{\varepsilon^{\prime}}{2}-\varepsilon\right)$, the minimization over states $\psi_{B}$ may be performed over pure states, and the outermost minimization is with respect to maps $\mathcal{N}_{A \rightarrow B}$ such that

$$
\operatorname{Tr}\left\{\left(\Pi_{\leq D}\right)_{R B}\left(\mathrm{id}_{R} \otimes \mathcal{N}_{A \rightarrow B}\right)\left(\varphi_{R A}^{\rho}\right)\right\} \geq 1-\varepsilon .
$$


Proof. First consider the type II error probability $\beta_{1-\varepsilon}\left(\varphi_{R A}^{\rho} \| \sigma_{R A}\right)$ defined in (20) for an arbitrary state $\sigma_{R A}$, as well as the quantity

$$
\max _{\psi_{B}} \beta_{1-\varepsilon^{\prime}}\left(\omega_{R B} \| \sigma_{R} \otimes \psi_{B}\right)=\max _{\psi_{B}} \min _{Q_{R B}}\left\{\operatorname{Tr}\left\{Q_{R B}\left(\sigma_{R} \otimes \psi_{B}\right)\right\}: 0 \leq Q_{R B} \leq I_{R B}, \operatorname{Tr}\left\{Q_{R B} \omega_{R B}\right\} \geq \varepsilon^{\prime}\right\}
$$

for some $\varepsilon^{\prime} \geq 2 \varepsilon$, where $\omega_{R B}$ is the final state of the protocol. We know from the minimax theorem that there is a state $\psi_{B}$ and a POVM element $Q_{R B}$ achieving the maximum and the minimum, respectively, in $\max _{\psi_{B}} \beta_{1-\varepsilon^{\prime}}\left(\omega_{R B} \| \sigma_{R} \otimes \psi_{B}\right)$, because the optimizations are over convex sets and the objective function is linear in the objects over which we are optimizing. Let $\psi_{B}^{*}$ and $Q_{R B}^{*}$ denote the state and POVM element, respectively, achieving the optimum. Now, from the definition of an $(M, D, \varepsilon)$ EA QRD code for $\left\{\mathcal{H}_{A}, \mathcal{H}_{B}, \rho, \Delta_{R B}\right\}$ (see Section 4.2), the following condition holds:

$$
\operatorname{Tr}\left\{\left(\Pi_{\leq D}\right)_{R B} \omega_{R B}\right\} \geq 1-\varepsilon .
$$

Let $\omega_{R B}^{\prime}$ denote the following state

$$
\omega_{R B}^{\prime}:=\frac{\sqrt{Q_{R B}^{*}} \omega_{R B} \sqrt{Q_{R B}^{*}}}{\operatorname{Tr}\left\{Q_{R B}^{*} \omega_{R B}\right\}} .
$$

By Lemma 3, we then have that

$$
\begin{aligned}
\operatorname{Tr}\left\{\left(\Pi_{\leq D}\right)_{R B} \omega_{R B}^{\prime}\right\} & \geq \operatorname{Tr}\left\{\left(\Pi_{\leq D}\right)_{R B} \omega_{R B}\right\}-\frac{1}{2}\left\|\omega_{R B}^{\prime}-\omega_{R B}\right\|_{1} \\
& \geq 1-\varepsilon-\frac{1}{2}\left\|\omega_{R B}^{\prime}-\omega_{R B}\right\|_{1} .
\end{aligned}
$$

We now compute an upper bound on $\frac{1}{2}\left\|\omega_{R B}^{\prime}-\omega_{R B}\right\|_{1}$. By letting $\left|\varphi^{\omega}\right\rangle_{R^{\prime} R B}$ be a particular purification of $\omega_{R B}$ and by exploiting Uhlmann's theorem, we have that

$$
\begin{aligned}
F\left(\omega_{R B}^{\prime}, \omega_{R B}\right)^{2} & \geq \frac{\left|\left\langle\left.\varphi^{\omega}\right|_{R^{\prime} R B}\left(I_{R^{\prime}} \otimes \sqrt{Q_{R B}^{*}}\right) \mid \varphi^{\omega}\right\rangle_{R^{\prime} R B}\right|^{2}}{\left\langle\left.\varphi^{\omega}\right|_{R^{\prime} R B}\left(I_{R^{\prime}} \otimes Q_{R B}^{*}\right) \mid \varphi^{\omega}\right\rangle_{R^{\prime} R B}} \\
& \geq\left\langle\left.\varphi^{\omega}\right|_{R^{\prime} R B}\left(I_{R^{\prime}} \otimes Q_{R B}^{*}\right) \mid \varphi^{\omega}\right\rangle_{R^{\prime} R B} \\
& \geq \varepsilon^{\prime} .
\end{aligned}
$$

Using the Fuchs-van-de-Graaf inequalities in (4), it follows that

$$
\begin{aligned}
\frac{1}{2}\left\|\omega_{R B}^{\prime}-\omega_{R B}\right\|_{1} & \leq \sqrt{1-F\left(\omega_{R B}^{\prime}, \omega_{R B}\right)^{2}} \\
& \leq \sqrt{1-\varepsilon^{\prime}} \\
& \leq 1-\frac{\varepsilon^{\prime}}{2}
\end{aligned}
$$

Substituting into (34) gives us that

$$
\begin{aligned}
\operatorname{Tr}\left\{\left(\Pi_{\leq D}\right)_{R B} \omega_{R B}^{\prime}\right\} & \geq 1-\varepsilon-\left(1-\frac{\varepsilon^{\prime}}{2}\right) \\
& =\frac{\varepsilon^{\prime}}{2}-\varepsilon,
\end{aligned}
$$


which finally gives that

$$
\begin{aligned}
\operatorname{Tr}\left\{\left(\Pi_{\leq D}\right)_{R B} \sqrt{Q_{R B}^{*}} \omega_{R B} \sqrt{Q_{R B}^{*}}\right\} & \geq \operatorname{Tr}\left\{Q_{R B}^{*} \omega_{R B}\right\}\left(\frac{\varepsilon^{\prime}}{2}-\varepsilon\right) \\
& \geq \varepsilon^{\prime}\left(\frac{\varepsilon^{\prime}}{2}-\varepsilon\right) \\
& =\varepsilon^{\prime \prime},
\end{aligned}
$$

by exploiting the definition in (33).

By the result in (35) and the fact that $\mathcal{N}_{A \rightarrow B}^{e a}$ from (30) is trace preserving, the following operator defines a particular POVM element $\Lambda_{R A}$ for which $\operatorname{Tr}\left\{\Lambda_{R A} \varphi_{R A}^{\rho}\right\} \geq \varepsilon^{\prime \prime}$ is true:

$$
\Lambda_{R A}=\left(\operatorname{id}_{R} \otimes \mathcal{N}_{A \rightarrow B}^{e a}\right)^{\dagger}\left(\sqrt{Q_{R B}^{*}}\left(\Pi_{\leq D}\right)_{R B} \sqrt{Q_{R B}^{*}}\right),
$$

where $\dagger$ indicates the adjoint of the $\operatorname{map}_{\operatorname{id}} \otimes \mathcal{N}_{A \rightarrow B}^{e a}$. Hence,

$$
\begin{aligned}
& \beta_{1-\varepsilon^{\prime \prime}}\left(\varphi_{R A}^{\rho} \| \sigma_{R A}\right) \\
& \leq \operatorname{Tr}\left\{\left(\operatorname{id}_{R} \otimes \mathcal{N}_{A \rightarrow B}^{e a}\right)^{\dagger}\left(\sqrt{Q_{R B}^{*}}\left(\Pi_{\leq D}\right)_{R B} \sqrt{Q_{R B}^{*}}\right) \sigma_{R A}\right\} \\
& =\operatorname{Tr}\left\{\left(\sqrt{Q_{R B}^{*}}\left(\Pi_{\leq D}\right)_{R B} \sqrt{Q_{R B}^{*}}\right)\left(\operatorname{id}_{R} \otimes\left(\mathcal{D}_{M T_{B} \rightarrow B} \circ \mathcal{E}_{A T_{A} \rightarrow M}\right)\right)\left(\sigma_{R A} \otimes \Psi_{T_{A} T_{B}}\right)\right\} \\
& \leq M \operatorname{Tr}\left\{\left(\sqrt{Q_{R B}^{*}}\left(\Pi_{\leq D}\right)_{R B} \sqrt{Q_{R B}^{*}}\right)\left(\operatorname{id}_{R} \otimes \mathcal{D}_{M T_{B} \rightarrow B}\right)\left(\sigma_{R} \otimes I_{M} \otimes \Psi_{T_{B}}\right)\right\} \\
& =M^{2} \operatorname{Tr}\left\{\left(\sqrt{Q_{R B}^{*}}\left(\Pi_{\leq D}\right)_{R B} \sqrt{Q_{R B}^{*}}\right)\left(\sigma_{R} \otimes \mathcal{D}_{M T_{B} \rightarrow B}\left(\pi_{M} \otimes \Psi_{T_{B}}\right)\right)\right\} \\
& \leq M^{2} \operatorname{Tr}\left\{Q_{R B}^{*}\left(\sigma_{R} \otimes \mathcal{D}_{M T_{B} \rightarrow B}\left(\pi_{M} \otimes \Psi_{T_{B}}\right)\right)\right\} \\
& \leq M^{2} \operatorname{Tr}\left\{Q_{R B}^{*}\left(\sigma_{R} \otimes \psi_{B}^{*}\right)\right\} \\
& =M^{2} \max _{\psi_{B}} \beta_{1-\varepsilon^{\prime}}\left(\omega_{R B} \| \sigma_{R} \otimes \psi_{B}\right) .
\end{aligned}
$$

The first inequality follows from the definition of $\beta_{1-\varepsilon^{\prime \prime}}\left(\varphi_{R A}^{\rho} \| \sigma_{R A}\right)$ and (36). The first equality follows by the definition of the adjoint map. The second inequality follows from the following operator inequality:

$$
\left(\mathrm{id}_{R} \otimes \mathcal{E}_{A T_{A} \rightarrow M}\right)\left(\sigma_{R A} \otimes \Psi_{T_{A} T_{B}}\right) \leq M\left(\sigma_{R} \otimes I_{M} \otimes \Psi_{T_{B}}\right) .
$$

which is an instance of the operator inequality $\rho_{A B} \leq|B|\left(\rho_{A} \otimes I_{B}\right)$ [7, 19]. The second equality follows from the definition $\pi_{M}:=I_{M} / M$. The third inequality follows from the operator inequality:

$$
\sqrt{Q_{R B}^{*}}\left(\Pi_{\leq D}\right)_{R B} \sqrt{Q_{R B}^{*}} \leq Q_{R B}^{*} .
$$

The last inequality follows because $\mathcal{D}_{M T_{B} \rightarrow B}\left(\pi_{M} \otimes \Psi_{T_{B}}\right)$ is a particular state and the expression should be optimized over pure states. That is, we can always take a spectral decomposition of $\mathcal{D}_{M T_{B} \rightarrow B}\left(\pi_{M} \otimes \Psi_{T_{B}}\right)$ as

$$
\mathcal{D}_{M T_{B} \rightarrow B}\left(\pi_{M} \otimes \Psi_{T_{B}}\right)=\sum_{z} p_{Z}(z)\left|\phi_{z}\right\rangle\left\langle\left.\phi_{z}\right|_{B},\right.
$$


and this leads to

$$
\begin{aligned}
& \operatorname{Tr}\left\{Q_{R B}^{*}\left(\sigma_{R} \otimes \mathcal{D}_{M T_{B} \rightarrow B}\left(\pi_{M} \otimes \Psi_{T_{B}}\right)\right)\right\} \\
& =\sum_{z} p_{Z}(z) \operatorname{Tr}\left\{Q_{R B}^{*}\left(\sigma_{R} \otimes\left|\phi_{z}\right\rangle\left\langle\left.\phi_{z}\right|_{B}\right)\right\}\right. \\
& \leq \max _{z} \operatorname{Tr}\left\{Q_{R B}^{*}\left(\sigma_{R} \otimes\left|\phi_{z}\right\rangle\left\langle\left.\phi_{z}\right|_{B}\right)\right\}\right. \\
& \leq \max _{\psi_{B}} \operatorname{Tr}\left\{Q_{R B}^{*}\left(\sigma_{R} \otimes \psi_{B}\right)\right\} .
\end{aligned}
$$

By taking a maximization over all states $\sigma_{R A}$, we arrive at the following bound:

$$
M \geq \max _{\sigma_{R A}} \min _{\psi_{B}} \sqrt{\frac{\beta_{1-\varepsilon^{\prime \prime}}\left(\varphi_{R A}^{\rho} \| \sigma_{R A}\right)}{\beta_{1-\varepsilon^{\prime}}\left(\omega_{R B} \| \sigma_{R} \otimes \psi_{B}\right)}} .
$$

Taking logarithms, we obtain that

$$
\log M \geq \frac{1}{2} \max _{\sigma_{R A}} \min _{\psi_{B}}\left[D_{H}^{1-\varepsilon^{\prime}}\left(\omega_{R B} \| \sigma_{R} \otimes \psi_{B}\right)-D_{H}^{1-\varepsilon^{\prime \prime}}\left(\varphi_{R A}^{\rho} \| \sigma_{R A}\right)\right] .
$$

Finally, we arrive at

$$
\log M \geq \frac{1}{2} \min _{\mathcal{N}_{A \rightarrow B}} \max _{\sigma_{R A}} \min _{\psi_{B}}\left[D_{H}^{1-\varepsilon^{\prime}}\left(\left(\operatorname{id}_{R} \otimes \mathcal{N}_{A \rightarrow B}\right)\left(\varphi_{R A}^{\rho}\right) \| \sigma_{R} \otimes \psi_{B}\right)-D_{H}^{1-\varepsilon^{\prime \prime}}\left(\varphi_{R A}^{\rho} \| \sigma_{R A}\right)\right] .
$$

by taking a minimization over all maps $\mathcal{N}_{A \rightarrow B}$ that meet the following excess-distortion probability constraint:

$$
\operatorname{Tr}\left\{\left(\Pi_{\leq D}\right)_{R B}\left(\operatorname{id}_{R} \otimes \mathcal{N}_{A \rightarrow B}\right)\left(\varphi_{R A}^{\rho}\right)\right\} \geq 1-\varepsilon
$$

By taking the state $\sigma_{R A}$ in the maximization in (31) to be equal to the purification $\varphi_{R A}^{\rho}$, we arrive at the following corollary of Proposition 7 ;

Corollary 8 Let $\rho$ be the density operator characterizing a quantum information source. For any $(M, D, \varepsilon)$ entanglement-assisted quantum rate distortion code for $\left\{\mathcal{H}_{A}, \mathcal{H}_{B}, \rho, \Delta_{R B}\right\}$, we have the following lower bound on its minimum qubit compression size:

$$
\log M^{*}\left(\rho, \Delta_{R B}, D, \varepsilon\right) \geq \frac{1}{2} \min _{\mathcal{N}_{A \rightarrow B}} \min _{\psi_{B}}\left[D_{H}^{1-\varepsilon^{\prime}}\left(\omega_{R B} \| \varphi_{R}^{\rho} \otimes \psi_{B}\right)-\log \frac{1}{\varepsilon^{\prime \prime}}\right]
$$

where $\varepsilon^{\prime} \geq 2 \varepsilon, \varepsilon^{\prime \prime}:=\varepsilon^{\prime}\left(\frac{\varepsilon^{\prime}}{2}-\varepsilon\right)$

$$
\omega_{R B}:=\left(\operatorname{id}_{R} \otimes \mathcal{N}_{A \rightarrow B}\right)\left(\varphi_{R A}^{\rho}\right),
$$

and the outermost minimization is with respect to maps $\mathcal{N}_{A \rightarrow B}$ such that

$$
\operatorname{Tr}\left\{\left(\Pi_{\leq D}\right)_{R B} \omega_{R B}\right\} \geq 1-\varepsilon .
$$




\subsection{An Alternative One-Shot Converse Bound}

This section details a quantum generalization of the converse theorem in [25]. The converse presented here lower bounds the minimum qubit compression size for any entanglement-assisted quantum rate distortion code, and it leads to a tight finite blocklength characterization of the entanglement-assisted quantum rate distortion function for an isotropic qubit source (see Section 10.1).

Proposition 9 Let $\rho$ be the density operator characterizing a quantum information source, and let $\left|\varphi^{\rho}\right\rangle_{R A}$ be a purification of it. For any $(M, D, \varepsilon)$ entanglement-assisted quantum rate distortion code for $\left\{\mathcal{H}_{A}, \mathcal{H}_{B}, \rho, \Delta_{R B}\right\}$, we have the following lower bound on its minimum qubit compression size:

$$
\log M^{*}\left(\rho, \Delta_{R B}, D, \varepsilon\right) \geq \frac{1}{2} \max _{\sigma_{R A}} \min _{\psi_{B}}\left[-\log \operatorname{Tr}\left\{\left(\Pi_{\leq D}\right)_{R B}\left(\sigma_{R} \otimes \psi_{B}\right)\right\}-D_{H}^{\varepsilon}\left(\varphi_{R A}^{\rho} \| \sigma_{R A}\right)\right] .
$$

Proof. We start with $\beta_{\varepsilon}\left(\varphi_{R A}^{\rho} \| \sigma_{R A}\right)$. From the definition of an $(M, D, \varepsilon)$ EA QRD code, (32) holds as in the previous proof. Thus, the following operator defines a particular POVM element $\Lambda_{R A}$ for which $\operatorname{Tr}\left\{\Lambda_{R A} \varphi_{R A}^{\rho}\right\} \geq 1-\varepsilon$ is true:

$$
\Lambda_{R A}=\left(\operatorname{id}_{R} \otimes \mathcal{N}_{A \rightarrow B}^{e a}\right)^{\dagger}\left(\left(\Pi_{\leq D}\right)_{R B}\right)
$$

where $\dagger$ indicates the adjoint of the map $\operatorname{id}_{R} \otimes \mathcal{N}_{A \rightarrow B}^{e a}$ and $\mathcal{N}_{A \rightarrow B}^{e a}$ is defined in (30). So $\beta_{\varepsilon}\left(\varphi_{R A}^{\rho} \| \sigma_{R A}\right)$ is upper bounded by

$$
\begin{aligned}
& \beta_{\varepsilon}\left(\varphi_{R A}^{\rho} \| \sigma_{R A}\right) \\
& \leq \operatorname{Tr}\left\{\left(\operatorname{id}_{R} \otimes \mathcal{N}_{A \rightarrow B}^{e a}\right)^{\dagger}\left(\left(\Pi_{\leq D}\right)_{R B}\right) \varphi_{R A}^{\sigma}\right\} \\
& =\operatorname{Tr}\left\{\left(\Pi_{\leq D}\right)_{R B}\left(\operatorname{id}_{R} \otimes \mathcal{D}_{M T_{B} \rightarrow B}\right)\left(\operatorname{id}_{R} \otimes \mathcal{E}_{A T_{A} \rightarrow M}\right)\left(\sigma_{R A} \otimes \Psi_{T_{A} T_{B}}\right)\right\} \\
& \leq M \operatorname{Tr}\left\{\left(\Pi_{\leq D}\right)_{R B}\left(\operatorname{id}_{R} \otimes \mathcal{D}_{M T_{B} \rightarrow B}\right)\left(\sigma_{R} \otimes I_{M} \otimes \Psi_{T_{B}}\right)\right\} \\
& =M^{2} \operatorname{Tr}\left\{\left(\Pi_{\leq D}\right)_{R B}\left(\sigma_{R} \otimes \mathcal{D}_{M T_{B} \rightarrow B}\left(\pi_{M} \otimes \Psi_{T_{B}}\right)\right)\right\} \\
& \leq M^{2} \max _{\psi_{B}} \operatorname{Tr}\left\{\left(\Pi_{\leq D}\right)_{R B}\left(\sigma_{R} \otimes \psi_{B}\right)\right\}
\end{aligned}
$$

These inequalities follow for very similar reasons as the inequalities in the proof of Proposition 7.

By rewriting (41) as

$$
\sqrt{\frac{\beta_{\varepsilon}\left(\varphi_{R A}^{\rho} \| \sigma_{R A}\right)}{\max _{\psi_{B}} \operatorname{Tr}\left\{\left(\Pi_{\leq D}\right)_{R B}\left(\sigma_{R} \otimes \psi_{B}\right)\right\}}} \leq M,
$$

optimizing the expression on the left with respect to the choice of $\sigma_{R A}$, and taking logarithms, we obtain the bound in the statement of the proposition.

This bound clearly applies to unassisted quantum rate distortion codes as well. This follows both operationally and from the fact that the bound applies when taking the systems $T_{A}$ and $T_{B}$ to be null. 


\subsection{Reduction to the Classical Kostina-Verdú Bound}

If the distortion observable is of the classical-classical type, then the analysis reduces to the classical case, and the above bound can be improved. Indeed, consider $\Delta_{R B}$ to have the form:

$$
\sum_{x}|x\rangle\left\langle\left. x\right|_{R} \otimes \Delta_{B}^{x}\right.
$$

where $\Delta_{B}^{x} \geq 0$ for all $x \in \mathcal{X}$. Then $\left(\Pi_{\leq D}\right)_{R B}$ takes the form $\sum_{x}|x\rangle\left\langle\left. x\right|_{R} \otimes\left(\Pi_{\leq D}^{x}\right)_{B}\right.$ where each $\left(\Pi_{\leq D}^{x}\right)_{B}$ is an excess-distortion projector corresponding to $\Delta_{B}^{x}$. We can bound $\beta_{\varepsilon}\left(\varphi_{R A}^{\rho} \| \sigma_{R A}\right)$ as

$$
\begin{aligned}
\beta_{\mathcal{E}}\left(\varphi_{R A}^{\rho} \| \sigma_{R A}\right) & \leq \operatorname{Tr}\left\{\left(\Pi_{\leq D}\right)_{R B}\left(\mathrm{id}_{R} \otimes \mathcal{D}\right)\left(\operatorname{id}_{R} \otimes \mathcal{E}\right)\left(\varphi_{R A}^{\sigma}\right)\right\} \\
& =\sum_{x} q_{\sigma}(x) \operatorname{Tr}\left\{\left(\Pi_{\leq D}^{x}\right)_{B}(\mathcal{D} \circ \mathcal{E})\left(\left|\psi^{x}\right\rangle\left\langle\left.\psi^{x}\right|_{A}\right)\right\}\right.
\end{aligned}
$$

where

$$
\begin{aligned}
\left|\psi^{x}\right\rangle_{A} & =\frac{1}{\sqrt{q_{\sigma}(x)}}\left(\langle x| \otimes I_{A}\right)\left|\varphi^{\sigma}\right\rangle_{R A}, \\
q_{\sigma}(x) & =\operatorname{Tr}\left\{\left(|x\rangle\langle x| \otimes I_{A}\right) \varphi_{R A}^{\sigma}\right\} .
\end{aligned}
$$

Continuing, we have the upper bound

$$
\beta_{\varepsilon}\left(\varphi_{R A}^{\rho} \| \sigma_{R A}\right) \leq M \max _{|\psi\rangle_{B}} \sum_{x} q_{\sigma}(x) \operatorname{Tr}\left\{\left(\Pi_{\leq D}^{x}\right)_{B} \psi_{B}\right\}
$$

and obtain the following bound analogous to that of Kostina and Verdú:

$$
M \geq \max _{\sigma \in \mathcal{D}\left(\mathcal{H}_{R A}\right)} \frac{\beta_{\varepsilon}\left(\varphi_{R A}^{\rho} \| \sigma_{R A}\right)}{\max _{\psi_{B}} \sum_{x} q_{\sigma}(x) \operatorname{Tr}\left\{\left(\Pi_{\leq D}^{x}\right)_{B} \psi_{B}\right\}} .
$$

\section{One-Shot Achievability Results via Channel Simulation}

In this section, we use known results on entanglement-assisted quantum channel simulation to find upper bounds on the minimum qubit compression size for an entanglement-assisted quantum rate distortion code that compresses a quantum information source $\left\{\rho, \mathcal{H}_{A}\right\}$. The basic idea is to simulate a quantum channel $\mathcal{N}_{A \rightarrow B}$ obeying a distortion constraint of the form

$$
\operatorname{Tr}\left\{\left(\Pi_{\leq D}\right)_{R B}\left(\operatorname{id}_{R} \otimes \mathcal{N}_{A \rightarrow B}\right)\left(\varphi_{R A}^{\rho}\right)\right\} \geq 1-\varepsilon .
$$

The quantum communication required in the simulation then constitutes an achievable compression size for the source.

For simplicity, fix the excess-distortion probability to be no larger than $\varepsilon$ and the distortion to be $D$, for a given distortion observable $\Delta_{R B}$. Then denote the minimum achievable code size as

$$
M^{*}:=M^{*}\left(\rho, \Delta_{R B}, D, \varepsilon\right),
$$

so that $\log M^{*}$ is the minimum qubit compression size. 
Suppose Alice has the source state $\rho \in \mathcal{D}\left(\mathcal{H}_{A}\right)$, a purification of which is given by $\varphi_{R A}^{\rho}$, with $R$ denoting the reference system. Additionally, Alice and Bob share entanglement which they can exploit to help them in their compression task. Now set $\varepsilon>0$ and choose an $\varepsilon_{1}>0$ such that $\varepsilon_{1}<\varepsilon$. To begin the simulation protocol, Alice locally applies an isometric extension $\mathcal{U}_{A \rightarrow A^{\prime} B}^{\mathcal{N}}$ of a CPTP map $\mathcal{N}_{A \rightarrow B}$ to the source state $\rho$, where $\mathcal{N}_{A \rightarrow B}$ satisfies

$$
\operatorname{Tr}\left\{\left(\Pi_{>D}\right)_{R B}\left(\operatorname{id}_{R} \otimes \mathcal{N}_{A \rightarrow B}\right)\left(\varphi_{R A}^{\rho}\right)\right\} \leq \varepsilon_{1},
$$

and $\left(\Pi_{>D}\right)_{R B}$ denotes the excess-distortion projector defined by (26). The resulting state, shared by Alice and the reference is given by

$$
\varphi_{R A^{\prime} B}^{\omega}=\left(\operatorname{id}_{R} \otimes \mathcal{U}_{A \rightarrow A^{\prime} B}^{\mathcal{N}}\right)\left(\varphi_{R A}^{\rho}\right)
$$

whose marginal state is

$$
\omega_{R B}=\operatorname{Tr}_{A^{\prime}}\left(\varphi_{R A^{\prime} B}^{\omega}\right)=\left(\mathrm{id}_{R} \otimes \mathcal{N}_{A \rightarrow B}\right)\left(\varphi_{R A}^{\rho}\right) .
$$

The next phase of the protocol is for Alice to transmit some quantum information to Bob, making use of the entanglement they share, to ensure that the final state shared between Bob and the reference system is $\left(\varepsilon-\varepsilon_{1}\right)$-close in trace distance to the state $\omega_{R B}$. One way for them to achieve this aim is via a one-shot $\left(\varepsilon-\varepsilon_{1}\right)$-error quantum state splitting protocol [7], in which the tripartite pure state $\varphi_{R A^{\prime} B}^{\omega}$, initially shared between the reference and Alice, is split between the reference, Alice, and Bob, such that Bob receives the system $B$ up to an error $\left(\varepsilon-\varepsilon_{1}\right)$. A state splitting protocol is a particular way to simulate a channel. The protocol consists of Alice applying local operations (denoted by the encoding CPTP map $\mathcal{E}$ ) on the systems in her possession (namely, the systems $A^{\prime} B$ and her share of the entanglement), sending qubits to Bob, and then Bob applying local operations on the system he receives and his share of the entanglement. Let $\log M(\rho, \mathcal{N})$ denote the minimum amount of quantum information that Alice needs to send to Bob when simulating the channel $\mathcal{N}$ on the state $\rho$. This quantum state splitting protocol simulates the output state of the quantum channel $\mathcal{N}$ on the source state $\rho$ (up to an error $\left(\varepsilon-\varepsilon_{1}\right)$ ), at Bob's end, and hence $(\mathcal{E}, \mathcal{D}, M(\rho, \mathcal{N}))$ constitutes a one-shot $\left(\varepsilon-\varepsilon_{1}\right)$-error channel simulation code.

Therefore, an upper bound on the minimum qubit compression size $\log M^{*}$ is given by

$$
\log M^{*} \leq \min _{\mathcal{N}_{A \rightarrow B}, \varepsilon_{1}}\left\{\log M(\rho, \mathcal{N}):(a),(b), 0<\varepsilon_{1}<\varepsilon\right\},
$$

where $(a)$ and $(b)$ denote the following conditions:

$$
\text { (a) }: \operatorname{Tr}\left\{\left(\Pi_{>D}\right)_{R B}\left(\operatorname{id}_{R} \otimes \mathcal{N}_{A \rightarrow B}\right)\left(\varphi_{R A}^{\rho}\right)\right\} \leq \varepsilon_{1},
$$

and

(b) : there exists a $(\mathcal{E}, \mathcal{D}, M(\mathcal{N}))$ one-shot $\left(\varepsilon-\varepsilon_{1}\right)$-error channel simulation code.

By applying Lemma 3, we obtain an upper bound on the excess-distortion probability for such a scheme:

$$
\begin{aligned}
& \operatorname{Tr}\left\{\left(\Pi_{>D}\right)_{R B}\left(\operatorname{id}_{R} \otimes \mathcal{D} \circ \mathcal{E} \circ \mathcal{U}_{A \rightarrow A^{\prime} B}^{\mathcal{N}}\right)\left(\varphi_{R A}^{\rho}\right)\right\} \\
& \leq \operatorname{Tr}\left\{\left(\Pi_{>D}\right)_{R B}\left(\operatorname{id}_{R} \otimes \mathcal{N}_{A \rightarrow B}\right)\left(\varphi_{R A}^{\rho}\right)\right\}+\left\|\left(\operatorname{id}_{R} \otimes \mathcal{D} \circ \mathcal{E} \circ \mathcal{U}_{A \rightarrow A^{\prime} B}^{\mathcal{N}}\right)\left(\varphi_{R A}^{\rho}\right)-\left(\operatorname{id}_{R} \otimes \mathcal{N}_{A \rightarrow B}\right)\left(\varphi_{R A}^{\rho}\right)\right\|_{1} \\
& \leq \varepsilon_{1}+\left(\varepsilon-\varepsilon_{1}\right)=\varepsilon .
\end{aligned}
$$


Remark 10 The results presented here and in prior work [32, 43, [26, 15] demonstrate that the tasks of channel simulation and rate distortion coding are related, but we should be careful not to conclude that they are the same task. In channel simulation, the criterion for a protocol to be successful is more stringent, in the sense that a third party should not be able to distinguish between the output of the actual channel and the simulated one if allowed to input arbitrary states (even entangled ones) to the channel. The demands of a rate-distortion protocol are not as stringent. For this task, a protocol is required to have an arbitrarily small excess distortion probability or meet an average distortion constraint, which depends on the distortion observable being employed. As we have seen in this section and in prior work [32, 43, 26, 15], a channel simulation protocol (specialized for tensor-power inputs) can be used for the task of rate distortion, but the opposite is not necessarily true. Furthermore, a channel simulation protocol might use more resources than are actually necessary to complete the rate-distortion task since the demands on it are more stringent. This overconsumption is negligible, for example, in the entanglement-assisted setting where an arbitrary amount of entanglement of an arbitrary type is allowed, but it is not so in the unassisted setting. In fact, one of the main open questions regarding quantum rate distortion is to characterize the unassisted quantum rate distortion function. The best known characterization employs channel simulation [15], and hence it can possibly be improved using a different method.

\subsection{Channel Simulation with the Help of an Arbitrary Entangled State}

First let us consider the situation in which the entanglement shared between Alice and Bob is allowed to be in an arbitrary form. In particular, we can allow them access to embezzling states [37, which is useful because they can generate any other entangled state from such a resource by acting only with local operations. Theorem III.10 of [7] (building upon prior work in [17, 1]) states that a one-shot $\left(\varepsilon-\varepsilon_{1}\right)$-error quantum state splitting protocol with a " $\delta$-ebit embezzling state" (for any $\delta>0$ ) can be achieved by quantum communication equal to

$$
\frac{1}{2} I_{\max }^{\delta^{\prime}}(B ; R)_{\omega}+2 \log \frac{1}{\delta^{\prime \prime}}+4+\log \log |B| .
$$

where $\varepsilon-\varepsilon_{1}=\left(\delta^{\prime \prime}+\delta^{\prime}+\delta \cdot \log |B|+|B|^{-\frac{1}{2}}\right), \delta^{\prime \prime}>0, \delta^{\prime}>0$, and $\omega_{R B}$ is the state defined by (50). In the above, $I_{\max }^{\delta^{\prime}}(B ; R)_{\omega}$ denotes the smooth max-information of $\omega_{R B}$ and is defined as in (15). As stated in Footnote 6 of [7], one can make the error $\varepsilon-\varepsilon_{1}$ arbitrarily small by enlarging the Hilbert space $B$ as needed to a space $B^{\prime}$ that contains $B$ as a subspace. This enlargement then increases the error term $\delta \cdot \log \left|B^{\prime}\right|$, but one can compensate for this by decreasing $\delta$ appropriately (taking a larger embezzling state). To simplify things a bit, we can just choose $\varepsilon_{1}=\delta^{\prime}=\delta^{\prime \prime}=\varepsilon / 5$, the enlarged space $B^{\prime}$ to have dimension at least $(5 / \varepsilon)^{2}$, and the term $\delta \cdot \log \left|B^{\prime}\right|$ to be no larger than $\varepsilon / 5$. Our conclusion is that a one-shot $4 \varepsilon / 5$-error quantum state splitting protocol can be achieved by quantum communication equal to

$$
\frac{1}{2} I_{\max }^{\varepsilon / 5}(B ; R)_{\omega}+2 \log (5 / \varepsilon)+4+\log \log \left(|B|+(5 / \varepsilon)^{2}\right),
$$

with the last term following from the fact that $\left|B^{\prime}\right|=\max \left(|B|,(5 / \varepsilon)^{2}\right) \leq|B|+(5 / \varepsilon)^{2}$.

Hence, if Alice and Bob share entanglement in the form of embezzling states, then the minimum achievable code size for an $(M, D, \varepsilon)$ entanglement-assisted quantum rate distortion code for 
$\left\{\mathcal{H}_{A}, \mathcal{H}_{B}, \rho, \Delta_{R B}\right\}$ is bounded from above as follows:

$$
\log M^{*} \leq \min _{\mathcal{N}_{A \rightarrow B}}\left\{\frac{1}{2} I_{\max }^{\varepsilon / 5}(B ; R)_{\omega}+2 \log (5 / \varepsilon)+4+\log \log \left(|B|+(5 / \varepsilon)^{2}\right):(a)\right\},
$$

where $\varepsilon_{1}$ in $(a)$ is equal to $\varepsilon / 5$.

\subsection{Channel Simulation with Maximally-Entangled States}

Now let us consider the situation in which the entanglement shared between Alice and Bob is restricted to be in the form of maximally entangled states. Note that in this case the one-shot quantum state-splitting protocol is the time-reversal of the one-shot fully-quantum Slepian-Wolf (FQSW) protocol [14. In the latter, Alice and Bob share a bipartite state, whose purification is held by an inaccessible reference, and the aim of the protocol is for Alice to send her system to Bob using as little quantum communication as possible, and at the same time generate entanglement with him. It can be viewed as a time-reversal of the quantum state splitting protocol because the resource, namely, entanglement, which is consumed in quantum state splitting, is generated in FQSW. An upper bound on the quantum communication cost for a one-shot $\left(\varepsilon-\varepsilon_{1}\right)$-error FQSW protocol, as obtained from Theorem 8 of [14], thus yields the following upper bound on $\log M(\rho, \mathcal{N})$ :

$$
\log M(\rho, \mathcal{N}) \leq \frac{1}{2}\left[H_{0}^{\delta}(B)_{\omega}-H_{\min }^{\delta}(B \mid R)_{\omega}\right]+\log \frac{1}{\delta^{\prime}}
$$

for some $\delta>0$, such that $\varepsilon=2 \sqrt{5 \delta^{\prime}}+2 \sqrt{\delta}$, and $\delta^{\prime}=\delta+\sqrt{4 \sqrt{\delta}-4 \delta}$. In the above, $\omega_{R B}$ denotes the state defined by (50), and $H_{0}^{\delta}(B)_{\omega}$ and $H_{\min }^{\delta}(B \mid R)_{\omega}$ are the smooth entropies of the state $\omega_{B}=\operatorname{Tr}_{R} \omega_{R B}$ and $\omega_{R B}$, defined as in (18) and (14) respectively. So if Alice and Bob share entanglement in the form of maximally entangled states, then

$$
\log M^{*} \leq \min _{\mathcal{N}_{A \rightarrow B}, \varepsilon_{1}}\left\{\frac{1}{2}\left[H_{0}^{\delta}(B)_{\omega}-H_{\min }^{\delta}(B \mid R)_{\omega}\right]+\log \frac{1}{\delta^{\prime}}:(a), 0<\varepsilon_{1}<\varepsilon\right\} .
$$

We should note that this bound is not as tight as the bound from the previous section, due to the following inequality [7]:

$$
I_{\max }(A ; B)_{\rho} \leq H_{0}(A)_{\rho}-H_{\min }(A \mid B)_{\rho} .
$$

Furthermore, the quantity on the right-hand side can become arbitrarily large when evaluated for particular states. However, if we restrict Alice and Bob to using maximally entangled states for entanglement assistance, then the bound in (59) is the best known bound.

\section{One-Shot Entanglement-Assisted Quantum Rate-Distortion The- orem}

This section unifies the converse bound from Corollary 8 and the achievability bound from Section 6.1 to establish a one-shot entanglement-assisted quantum rate-distortion theorem. The following theorem shows that the upper and lower bounds on the minimum qubit compression size for an entanglement-assisted quantum rate-distortion code can both be expressed in terms of the 
same smooth entropic quantity, namely, the smooth max-information, up to logarithmic correction terms 6

Theorem 11 Let $\rho \in \mathcal{D}\left(\mathcal{H}_{A}\right)$ be the density operator characterizing a quantum information source, and let $\left|\varphi^{\rho}\right\rangle_{R A}$ be a purification of it. For any $(M, D, \varepsilon)$ entanglement-assisted quantum rate distortion code for $\left\{\mathcal{H}_{A}, \mathcal{H}_{B}, \rho, \Delta_{R B}\right\}$, we have the following bounds on its minimum qubit compression size:

$$
\begin{aligned}
\min _{\mathcal{N}_{A \rightarrow B}}\{ & \left.\frac{1}{2} I_{\max }^{\varepsilon / 5}(B ; R)_{\omega}+\chi_{1}: \operatorname{Tr}\left(\left(\Pi_{\leq D}\right)_{R B} \omega_{R B}\right) \geq 1-\varepsilon / 5\right\} \geq \\
& \log M^{*}\left(\rho, \Delta_{R B}, D, \varepsilon\right) \geq \min _{\mathcal{N}_{A \rightarrow B}}\left\{\frac{1}{2} I_{\max }^{2 \sqrt{2 \varepsilon^{\prime}}}(B ; R)_{\omega}-\chi_{2}: \operatorname{Tr}\left(\left(\Pi_{\leq D}\right)_{R B} \omega_{R B}\right) \geq 1-\varepsilon\right\},
\end{aligned}
$$

where

$$
\begin{aligned}
\omega_{R B} & :=\left(\operatorname{id}_{R} \otimes \mathcal{N}_{A \rightarrow B}\right) \varphi_{R A}^{\rho}, \\
\chi_{1} & :=2 \log (5 / \varepsilon)+4+\log \log \left(|B|+(5 / \varepsilon)^{2}\right) \\
\chi_{2} & :=\frac{1}{2} \log \left(\left(\frac{1}{\varepsilon^{\prime}}+\frac{1}{1-\sqrt{2 \varepsilon^{\prime}}}\right)\left(\frac{1}{\varepsilon^{\prime} / 2-\varepsilon}\right)\right),
\end{aligned}
$$

$\varepsilon^{\prime} \geq 2 \varepsilon$, and $\mathcal{N}_{A \rightarrow B}$ is a CPTP map from $\mathcal{D}\left(\mathcal{H}_{A}\right)$ to $\mathcal{D}\left(\mathcal{H}_{B}\right)$.

Remark 12 In the special case of (almost) lossless quantum data compression, i.e., $D=0$ and $\Delta_{R B}=I_{R B}-\left|\varphi_{R B}\right\rangle\left\langle\varphi_{R B}\right|$ (with $\varphi_{R B}:=\varphi^{\rho}$ a purification of $\rho_{A}$ and $\mathcal{H}_{A}$ isomorphic to $\mathcal{H}_{B}$ ), it is known that the minimum qubit compression size is given by $H_{0}^{\epsilon}\left(\rho_{A}\right)$. This, together with Theorem 10, gives an operational proof that $I_{\max }^{\varepsilon^{\prime}}(A: R)_{\varphi}$, for $\varphi=\varphi_{A R}^{\rho}$ a pure state, is approximately (up to additive terms of the form $\chi_{1}$ and $\chi_{2}$, and some appropriately chosen $\varepsilon^{\prime}>0$ ) equal to $H_{0}^{\varepsilon}\left(\rho_{A}\right)$.

Remark 13 The above converse bound and achievability result can be applied to (unassisted) oneshot rate distortion in the purely classical setting. To do so, pick a distortion observable of classicalclassical type as in (11) and consider classical information sources (diagonal in the same basis as the distortion observable). The converse bound, which includes the possibility of entanglement assistance, also bounds the unassisted case. Channel simulation in the achievability argument nominally requires the use of embezzling states, but for classical channels this can be reduced to randomness shared between sender and receiver, as for the case of quantum-to-classical channels (measurements) in [8]. However, the channel simulation is only used to output a state $\omega_{R B}$ which satisfies the constraint on the excess distortion probability, $\operatorname{Tr}\left(\left(\Pi_{>D}\right)_{R B} \omega_{R B}\right) \leq \varepsilon$. Since this constraint is linear, we may interpret it as the average constraint for the different states $\omega_{R B}^{i}$ resulting from the shared randomness $i$, and we are free to pick the best value (least excess distortion probability) $i$.

\footnotetext{
${ }^{6}$ However, note that it is possible to provide a similar characterization in terms of the hypothesis testing relative entropy $D_{H}^{1-\varepsilon}$ or the alternative smooth max-information (defined in (68), due to the relation between these quantities and the smooth max-information.
} 
Proof of Theorem 11. The upper bound on $\log M^{*}$ follows readily from the result in (57). So we focus on establishing the lower bound on $\log M^{*}$. Corollary 8 establishes (38) as a lower bound on $\log M^{*}$, and we find that

$$
\begin{aligned}
\log M^{*} & \geq \frac{1}{2}\left[\min _{\mathcal{N}_{A \rightarrow B}} \min _{\sigma_{B}} D_{H}^{1-\varepsilon^{\prime}}\left(\omega_{R B} \| \varphi_{R}^{\rho} \otimes \sigma_{B}\right)-\log \frac{1}{\varepsilon^{\prime \prime}}\right] \\
& \geq \frac{1}{2}\left[\min _{\mathcal{N}_{A \rightarrow B}} \min _{\sigma_{B}} D_{\max }^{\sqrt{2 \varepsilon^{\prime}}}\left(\omega_{R B} \| \varphi_{R}^{\rho} \otimes \sigma_{B}\right)-\log \left(\frac{1}{\varepsilon^{\prime} / 2-\varepsilon}\right)\right] \\
& \geq \frac{1}{2}\left[\min _{\mathcal{N}_{A \rightarrow B}} \min _{\sigma_{B}} \min _{\tau_{R}} \min _{R B} D_{B^{\sqrt{2 \varepsilon^{\prime}}}}\left(\omega_{R B}\right)\right. \\
& \left.D_{\max }\left(\widetilde{\omega}_{R B} \| \tau_{R} \otimes \sigma_{B}\right)-\log \left(\frac{1}{\varepsilon^{\prime} / 2-\varepsilon}\right)\right] \\
& =\frac{1}{2}\left[\min _{\mathcal{N}_{A \rightarrow B}} \widetilde{I}_{\max }^{\sqrt{2 \varepsilon^{\prime}}}(B ; R)_{\omega}-\log \left(\frac{1}{\varepsilon^{\prime} / 2-\varepsilon}\right)\right] .
\end{aligned}
$$

The first inequality exploits Corollary $\left[\right.$, but using a minimization over mixed states $\sigma_{B}$ (recall that even if the minimization is defined to be over mixed states, the optimizing state will be pure). The second inequality follows from the relation in Lemma 1 between the hypothesis testing relative entropy and the smooth max-relative entropy. The third inequality follows by taking a further minimization over states $\tau_{R}$ and by recalling the definition of the smooth max-relative entropy. The equality follows by defining the alternative smooth max-information as [10, 11$]$

$$
\widetilde{I}_{\max }^{\varepsilon}(R ; B)_{\omega}:=\min _{\widetilde{\omega}_{R B} \in B^{\varepsilon}\left(\omega_{R B}\right)} \widetilde{I}_{\max }(R ; B)_{\widetilde{\omega}},
$$

where

$$
\widetilde{I}_{\max }(R ; B)_{\omega}:=\min _{\sigma_{R} \in \mathcal{D}\left(\mathcal{H}_{R}\right)} \min _{\tau_{B} \in \mathcal{D}\left(\mathcal{H}_{B}\right)} D_{\max }\left(\omega_{R B} \| \sigma_{R} \otimes \tau_{B}\right)
$$

Now consider the following relation between the alternative smooth max-information $\widetilde{I}_{\max }^{\varepsilon^{\prime \prime \prime}}(B ; R)_{\omega}$ and the smooth max-information $I_{\max }^{\varepsilon^{\prime \prime}+\varepsilon^{\prime \prime \prime}}(B ; R)_{\omega}$ from Lemma 4.2.1 of [10]: for any $\varepsilon^{\prime \prime}>0$ and any $\varepsilon^{\prime \prime \prime} \geq 0$,

$$
I_{\max }^{\varepsilon^{\prime \prime}+\varepsilon^{\prime \prime \prime}}(B ; R)_{\omega} \leq \widetilde{I}_{\max }^{\varepsilon^{\prime \prime \prime}}(B ; R)_{\omega}+\log \left(\frac{2}{\left(\varepsilon^{\prime \prime}\right)^{2}}+\frac{1}{1-\varepsilon^{\prime \prime \prime}}\right) .
$$

Choosing $\varepsilon^{\prime \prime}=\sqrt{2 \varepsilon^{\prime}}$ and $\varepsilon^{\prime \prime \prime}=\sqrt{2 \varepsilon^{\prime}}$ and applying the above relation, we find that the RHS of (67) is larger than

$$
\frac{1}{2}\left[\min _{\mathcal{N}_{A \rightarrow B}} I_{\max }^{2 \sqrt{2 \varepsilon^{\prime}}}(B ; R)_{\omega}-\log \left(\left(\frac{1}{\varepsilon^{\prime}}+\frac{1}{1-\sqrt{2 \varepsilon^{\prime}}}\right)\left(\frac{1}{\varepsilon^{\prime} / 2-\varepsilon}\right)\right)\right],
$$

giving us the lower bound on $\log M^{*}$ stated in the theorem.

\section{Finite Blocklength Quantum Rate Distortion Coding}

One of the most important settings for quantum rate distortion theory is the independent and identically distributed (i.i.d.) setting with an average symbol-wise distortion observable. In this case, the source is specified as $n$ copies of some density operator $\rho_{A}$, where $n$ is some finite positive integer, and it is helpful to consider a purification $\left|\varphi^{\rho}\right\rangle_{R A}^{\otimes n}$ of the source. In this case, one considers block codes of length $n$ defined by an encoding map $\mathcal{E}_{A^{n} \rightarrow M^{n}}: \mathcal{D}\left(\mathcal{H}_{A}^{\otimes n}\right) \mapsto \mathcal{D}\left(\mathcal{H}_{M}^{\otimes n}\right)$, and a decoding map $\mathcal{D}_{M^{n} \rightarrow B^{n}}: \mathcal{D}\left(\mathcal{H}_{M}^{\otimes n}\right) \mapsto \mathcal{D}\left(\mathcal{H}_{B}^{\otimes n}\right)$. The relevant distortion observable in this scenario is the average symbol-wise distortion observable which is defined as follows: 
Definition 14 (Average symbol-wise distortion observable) Given a single-symbol distortion observable $\Delta_{R B}$, we can define an average symbol-wise distortion observable $\bar{\Delta}_{R^{n} B^{n}}$ acting on $n$ symbols as follows:

$$
\bar{\Delta}_{R^{n} B^{n}}:=\frac{1}{n} \sum_{i=1}^{n}\left(I^{\otimes(i-1)}\right)_{R_{1}^{i-1} B_{1}^{i-1}} \otimes \Delta_{R_{i} B_{i}} \otimes\left(I^{\otimes(n-i)}\right)_{R_{i+1}^{n} B_{i+1}^{n}} .
$$

where $R_{1}^{i-1}:=R_{1} \cdots R_{i-1}, \quad R_{i+1}^{n}:=R_{i+1} \cdots R_{n}$, with a similar convention for $B_{1}^{i-1}$ and $B_{i+1}^{n}$.

The following lemma gives a particular form for the spectral decomposition of $\bar{\Delta}_{R^{n} B^{n}}$, which in turn leads to a specification of the average symbol-wise excess-distortion projector, the latter being an operator defined as follows. If the spectral decomposition of $\bar{\Delta}_{R^{n} B^{n}}$ is given by $\bar{\Delta}_{R^{n} B^{n}}=$ $\sum_{i} \lambda_{i} P_{i}^{n}$, then for any distortion $D>0$, the average symbol-wise excess-distortion projector is given by $\left(\bar{\Pi}_{>D}\right)_{R^{n} B^{n}}:=\sum_{i: \lambda_{i}>D} P_{i}^{n}$.

Lemma 15 The average symbol-wise distortion observable $\bar{\Delta}_{R^{n} B^{n}}$ has the following spectral decomposition:

$$
\bar{\Delta}_{R^{n} B^{n}}=\sum_{z^{n}} \bar{d}_{z^{n}}\left|\phi_{z^{n}}\right\rangle\left\langle\phi_{z^{n}}\right|
$$

where

$$
\begin{aligned}
z^{n} & :=\left(z_{1}, z_{2}, \ldots, z_{n}\right), \\
\bar{d}_{z^{n}} & :=\frac{1}{n} \sum_{i=1}^{n} d_{z_{i}}, \\
\left|\phi_{z^{n}}\right\rangle & :=\left|\phi_{z_{1}}\right\rangle \otimes \cdots \otimes\left|\phi_{z_{n}}\right\rangle,
\end{aligned}
$$

and $d_{z_{i}}$ and $\left|\phi_{z_{i}}\right\rangle$ are defined through the spectral decomposition of $\Delta_{R_{i} B_{i}}$.

The decomposition in (73) implies that the average symbol-wise excess-distortion projector can be written as

$$
\left(\bar{\Pi}_{>D}\right)_{R^{n} B^{n}}=\sum_{z^{n}: \bar{d}_{z^{n}}>D}\left|\phi_{z^{n}}\right\rangle\left\langle\phi_{z^{n}}\right|
$$

Proof. One can easily check that $\left|\phi_{z^{n}}\right\rangle$ is an eigenvector of $\bar{\Delta}_{R^{n} B^{n}}$ with eigenvalue $\bar{d}_{z^{n}}$. Since the orthonormal basis $\left\{\left|\phi_{z^{n}}\right\rangle\right\}$ spans the whole support of $\bar{\Delta}_{R^{n} B^{n}}$, this eigenvector-eigenvalue relation implies that $\bar{\Delta}_{R^{n} B^{n}}$ has the spectral decomposition as given in the statement of the lemma. The form of the average symbol-wise excess-distortion projector follows readily from its definition and the decomposition in (73).

Remark 16 As remarked in Refs. [16, 40], the classical case emerges as a special case in the distortion observable framework. In the classical case, the distortion observable is taken to be of the classical-classical type:

$$
\Delta_{R B}=\sum_{x, y} d(x, y)|x\rangle\left\langle\left. x\right|_{R} \otimes \mid y\right\rangle\left\langle\left. y\right|_{B},\right.
$$

for some distortion measure $d(x, y)$ and orthonormal bases $\{|x\rangle\},\{|y\rangle\}$. By applying Lemma 15, the average symbol-wise distortion observable becomes

$$
\bar{\Delta}_{R^{n} B^{n}}=\sum_{x^{n}, y^{n}} \bar{d}\left(x^{n}, y^{n}\right)\left|x^{n}\right\rangle\left\langle\left. x^{n}\right|_{R^{n}} \otimes \mid y^{n}\right\rangle\left\langle\left. y^{n}\right|_{B^{n}},\right.
$$


where

$$
\bar{d}\left(x^{n}, y^{n}\right)=\frac{1}{n} \sum_{i=1}^{n} d\left(x_{i}, y_{i}\right) .
$$

Analogously to the one-shot case described in Section 4.2, we define an $\left(M_{n}, D, \varepsilon\right)$ entanglementassisted quantum rate distortion code of blocklength $n$ as follows.

Definition 17 An $\left(M_{n}, D, \varepsilon\right)$ entanglement-assisted quantum rate distortion (EA QRD) code for $\left\{\mathcal{H}_{A}^{\otimes n}, \mathcal{H}_{B}^{\otimes n}, \rho^{\otimes n}, \bar{\Delta}_{R^{n} B^{n}}\right\}$ is a code with $\left|\mathcal{H}_{M}^{\otimes n}\right|=M_{n}$ such that

$$
\operatorname{Tr}\left\{\left(\bar{\Pi}_{>D}\right)_{R^{n} B^{n}}\left(\operatorname{id}_{R^{n}} \otimes\left(\mathcal{N}_{A^{n} \rightarrow B^{n}}^{e a}\right)\right)\left(\varphi_{R A}^{\rho}\right)^{\otimes n}\right\} \leq \varepsilon .
$$

where

$$
\mathcal{N}_{A^{n} \rightarrow B^{n}}^{e a}\left(\sigma_{A^{n}}\right):=\left(\mathcal{D}_{M^{n} T_{B} \rightarrow B} \circ \mathcal{E}_{A^{n} T_{A} \rightarrow M^{n}}\right)\left(\sigma_{A^{n}} \otimes \Psi_{T_{A} T_{B}}\right),
$$

The corresponding minimum achievable code size, denoted as $M_{n}^{*}\left(\rho^{\otimes n}, \bar{\Delta}_{R^{n} B^{n}}, D, \varepsilon\right)$, is the minimum value of $M$ such that there exists an $\left(M_{n}, D, \varepsilon\right) E A$ QRD code of blocklength $n$.

We define the mean distortion of $n$ copies of the source state, $\rho^{\otimes n}$, under any CPTP map $\mathcal{N}_{A^{n} \rightarrow B^{n}}$, analogously to the one-shot case, but in terms of the corresponding average symbol-wise distortion observable $\bar{\Delta}_{R^{n} B^{n}}$ :

$$
\delta_{\text {mean }}^{(n)}\left(\rho, \mathcal{N}_{A^{n} \rightarrow B^{n}}, \Delta_{R B}\right):=\operatorname{Tr}\left(\bar{\Delta}_{R^{n} B^{n}} \omega_{R^{n} B^{n}}\right),
$$

where $\omega_{R^{n} B^{n}}:=\left(\operatorname{id}_{R^{n}} \otimes \mathcal{N}_{A^{n} \rightarrow B^{n}}\right)\left(\varphi_{R A}^{\rho}\right)^{\otimes n}$. In particular, such a map induces the following mean distortion on the $i^{\text {th }}$ subsystems $R_{i} B_{i}$ :

$$
\delta_{\text {mean }}\left(\rho, \mathcal{N}_{A^{n} \rightarrow B^{n}}^{(i)}, \Delta_{R B}\right):=\operatorname{Tr}\left(\Delta_{R_{i} B_{i}} \omega_{R_{i} B_{i}}\right),
$$

where $\omega_{R_{i} B_{i}}$ is the reduced state of $\omega_{R^{n} B^{n}}$ on the subsystems $R_{i} B_{i}$, and $\mathcal{N}_{A^{n} \rightarrow B^{n}}^{(i)}$ denotes the marginal operation on these systems and is given by

$$
\mathcal{N}_{A^{n} \rightarrow B^{n}}^{(i)}\left(\rho^{\otimes n}\right):=\operatorname{Tr}_{A_{1}, A_{2}, \ldots, A_{i-1}, A_{i+i}, \ldots, A_{n}}\left(\mathcal{N}_{A^{n} \rightarrow B^{n}}\left(\rho^{\otimes n}\right)\right)
$$

Lemma 18 Fix $\varepsilon>0, D>0$ and consider a rate distortion observable $\Delta_{R B}$. If $\mathcal{N}_{A^{n} \rightarrow B^{n}}$ is a CPTP map acting on $\rho^{\otimes n}$ such that

$$
\operatorname{Tr}\left(\left(\bar{\Pi}_{\leq D}\right)_{R^{n} B^{n}} \omega_{R^{n} B^{n}}\right) \geq 1-\varepsilon,
$$

where $\omega_{R^{n} B^{n}}:=\left(i d_{R^{n}} \otimes \mathcal{N}_{A^{n} \rightarrow B^{n}}\right)\left(\varphi_{R A}^{\rho}\right)^{\otimes n}$, then the corresponding mean distortion satisfies the bound

$$
\delta_{\text {mean }}^{(n)}\left(\rho, \mathcal{N}_{A^{n} \rightarrow B^{n}}, \Delta_{R B}\right) \leq D+d_{\max } \varepsilon
$$

where $d_{\max }$ denotes the maximum eigenvalue of the distortion observable.

Proof. This follows directly from the definitions $(72)$ and $(77)$ of $\bar{\Delta}_{R^{n} B^{n}}$ and $\left(\bar{\Pi}_{\leq D}\right)_{R^{n} B^{n}}$, and is analogous to the proof of Lemma 6 , 


\subsection{Channel Simulation, Quantum Rate Distortion Coding, and Excess-Distortion Probability}

Lemma 1 of [15] shows that a channel simulation protocol can always be used for quantum rate distortion coding with an average symbol-wise distortion constraint, whenever the simulated channel meets a mean single-symbol distortion constraint 7 The following lemma is a counterpart to that result - Lemma 19 shows that a channel simulation protocol can always be used for quantum rate distortion coding under a symbol-wise excess-distortion probability constraint, whenever the simulated channel meets a mean single-symbol distortion constraint:

Lemma 19 Fix $\varepsilon_{\operatorname{sim}}, \delta>0$ and $D \geq 0$. Let $\Delta_{R B}$ be a distortion observable such that $\left\|\Delta_{R B}\right\|_{\infty}=$ $d_{\max }<\infty$. Let $\rho_{A}$ be a state with purification $\left|\varphi^{\rho}\right\rangle_{R A}$ and $\mathcal{N}_{A \rightarrow B}$ be a quantum channel such that

$$
\operatorname{Tr}\left\{\Delta_{R B} \omega_{R B}\right\} \leq D-\delta,
$$

where $\omega_{R B}:=\left(\operatorname{id}_{R} \otimes \mathcal{N}_{A \rightarrow B}\right)\left(\varphi_{R A}^{\rho}\right)$ and $\operatorname{supp}\left(\omega_{R B}\right) \subseteq \operatorname{supp}\left(\Delta_{R B}\right)$. Furthermore, let $\mathcal{F}_{n}: \mathcal{D}\left(\mathcal{H}_{A}^{\otimes n}\right) \mapsto$ $\mathcal{D}\left(\mathcal{H}_{B}^{\otimes n}\right)$ denote a quantum operation, such that

$$
\frac{1}{2}\left\|\sigma_{R^{n} B^{n}}-\omega_{R B}^{\otimes n}\right\|_{1} \leq \varepsilon_{\text {sim }},
$$

where

$$
\sigma_{R^{n} B^{n}}:=\left(\operatorname{id}_{R^{n}} \otimes \mathcal{F}_{n}\right)\left(\left(\varphi_{R A}^{\rho}\right)^{\otimes n}\right) .
$$

Then the average symbol-wise excess-distortion probability satisfies the following bound:

$$
\operatorname{Tr}\left\{\left(\bar{\Pi}_{>D}\right)_{R^{n} B^{n}} \sigma_{R^{n} B^{n}}\right\} \leq \exp \left\{-2 n \delta^{2} / d_{\max }^{2}\right\}+\varepsilon_{\text {sim }} .
$$

Proof. Let $Z$ be a random variable with the following distribution:

$$
p_{Z}(z):=\left\langle\phi_{z}\left|\omega_{R B}\right| \phi_{z}\right\rangle,
$$

where we recall that $\Delta_{R B}=\sum_{z} d_{z}\left|\phi_{z}\right\rangle\left\langle\phi_{z}\right|$. Let the $n^{\text {th }}$ i.i.d. extension $Z^{n}$ of $Z$ have the distribution:

$$
p_{Z^{n}}\left(z^{n}\right):=\left\langle\phi_{z^{n}}\left|\omega_{R B}^{\otimes n}\right| \phi_{z^{n}}\right\rangle
$$

Then the first condition in the statement of the lemma is equivalent to

$$
\operatorname{Tr}\left\{\Delta_{R B} \omega_{R B}\right\}=\sum_{z} d_{z} p_{Z}(z)=\mathbb{E}_{Z}\left\{d_{Z}\right\} \leq D-\delta .
$$

Also, observe from the i.i.d. assumption that $\mathbb{E}_{Z}\left\{d_{Z}\right\}=\mathbb{E}_{Z^{n}}\left\{\bar{d}_{Z^{n}}\right\}$, so that

$$
\mathbb{E}_{Z^{n}}\left\{\bar{d}_{Z^{n}}\right\}+\delta \leq D
$$

Using the spectral decomposition in (77) and the definition of $p_{Z^{n}}$, we can then write

$$
\operatorname{Tr}\left\{\left(\bar{\Pi}_{>D}\right)_{R^{n} B^{n}} \omega_{R B}^{\otimes n}\right\}=\sum_{z^{n}: \bar{d}_{z^{n}}>D} p_{Z^{n}}\left(z^{n}\right) .
$$

\footnotetext{
${ }^{7}$ Lemma 1 of 15 w was proved for the entanglement fidelity based distortion measure, but a quick inspection of its proof reveals that the lemma holds for an arbitrary distortion observable.
} 
An exponentially decreasing tail bound on $\operatorname{Tr}\left\{\left(\bar{\Pi}_{>D}\right)_{R^{n} B^{n}} \omega_{R B}^{\otimes n}\right\}$ follows by appealing to Hoeffding's inequality [23, 12]:

$$
\begin{aligned}
\sum_{z^{n}: \bar{d}_{z^{n}}>D} p_{Z^{n}}\left(z^{n}\right) & =\operatorname{Pr}_{Z^{n}}\left\{\bar{d}_{Z^{n}}>D\right\} \\
& \leq \operatorname{Pr}_{Z^{n}}\left\{\bar{d}_{Z^{n}}>\mathbb{E}_{Z^{n}}\left\{\bar{d}_{Z^{n}}\right\}+\delta\right\} \\
& \leq \exp \left\{-2 n \delta^{2} / d_{\max }^{2}\right\} .
\end{aligned}
$$

We obtain the statement of the lemma by appealing to Lemma 3 and the above inequality:

$$
\begin{aligned}
\operatorname{Tr}\left\{\left(\bar{\Pi}_{>D}\right)_{R^{n} B^{n}} \sigma_{R^{n} B^{n}}\right\} & \leq \operatorname{Tr}\left\{\left(\bar{\Pi}_{>D}\right)_{R^{n} B^{n}} \omega_{R B}^{\otimes n}\right\}+\frac{1}{2}\left\|\sigma_{R^{n} B^{n}}-\omega_{R B}^{\otimes n}\right\|_{1} \\
& \leq \exp \left\{-2 n \delta^{2} / d_{\max }^{2}\right\}+\varepsilon_{\text {sim. }}
\end{aligned}
$$

Remark 20 In much of the prior work on quantum rate distortion theory, the channel simulation method was used to prove achievability for rate distortion coding with a mean distortion constraint in a variety of scenarios [26, 15, 16, 40]. The above lemma demonstrates that all of these channel simulation methods can be extended to achieve rate distortion coding with an excess-distortion probability constraint.

\subsection{Average Symbol-Wise Entanglement Fidelity Distortion Observable}

A particular example of an average symbol-wise distortion measure is the entanglement fidelity based distortion measure that Barnum introduced [3]. The distortion observable corresponding to it is taken from the average symbol-wise entanglement fidelity:

$$
\bar{\Delta}_{R^{n} B^{n}}:=\frac{1}{n} \sum_{i=1}^{n}\left(I^{\otimes(i-1)}\right)_{R_{1}^{i-1} B_{1}^{i-1}} \otimes\left(I_{R_{i} B_{i}}-\left|\varphi^{\rho}\right\rangle\left\langle\left.\varphi^{\rho}\right|_{R_{i} B_{i}}\right) \otimes\left(I^{\otimes(n-i)}\right)_{R_{i+1}^{n} B_{i+1}^{n}} .\right.
$$

We can think of this distortion observable as being analogous to a Hamiltonian that assigns an energy penalty of one on average if the output state is orthogonal to $\left|\varphi^{\rho}\right\rangle\left\langle\left.\varphi^{\rho}\right|_{R B}\right.$. Thus, the above distortion observable is a quantum analog of the classical Hamming distortion measure. We can expand the distortion observable $\bar{\Delta}_{R^{n} B^{n}}$ by making the following assignments:

$$
\begin{aligned}
& \Pi_{0}:=\left|\varphi^{\rho}\right\rangle\left\langle\left.\varphi^{\rho}\right|_{R B},\right. \\
& \Pi_{1}:=I_{R B}-\left|\varphi^{\rho}\right\rangle\left\langle\left.\varphi^{\rho}\right|_{R B} .\right.
\end{aligned}
$$

By applying the spectral decomposition in (73), we arrive at

$$
\bar{\Delta}_{R^{n} B^{n}}=\sum_{j=1}^{n} \frac{j}{n}\left[\sum_{x^{n} \in\{0,1\}^{n}: \operatorname{wt}\left(x^{n}\right)=j} \Pi_{x^{n}}\right]
$$

where

$$
\Pi_{x^{n}}:=\Pi_{x_{1}} \otimes \cdots \otimes \Pi_{x_{n}}
$$


and $\operatorname{wt}\left(x^{n}\right)$ is equal to the Hamming weight of the string $x^{n}$. Thus, the analogy with the classical Hamming weight distortion measure becomes clear: A reproduction of the quantum source at the output is given an average penalty proportional to the number of terms in the tensor product that are orthogonal to $\left|\varphi^{\rho}\right\rangle\left\langle\left.\varphi^{\rho}\right|_{R B}\right.$.

By applying Lemma 15] again, we can determine the form of the excess-distortion observable $\left(\bar{\Pi}_{>D}\right)_{R^{n} B^{n}}$ corresponding to $\bar{\Delta}_{R^{n} B^{n}}$, with $0 \leq D \leq 1$. Since two projectors $\Pi_{x^{n}}$ and $\Pi_{y^{n}}$ with $x^{n}, y^{n} \in\{0,1\}^{n}$ are orthogonal whenever $x^{n} \neq y^{n}$, by using (26) and (89), we can write

$$
\left(\bar{\Pi}_{>D}\right)_{R^{n} B^{n}}=\sum_{j \in\{1, \ldots, n\}: j / n>D}\left[\sum_{x^{n} \in\{0,1\}^{n}: \operatorname{wt}\left(x^{n}\right)=j} \Pi_{x^{n}}\right] .
$$

\section{First-Order Convergence for a Memoryless Quantum Source}

Consider the case in which Alice has $n>1$ copies of the source state $\rho \in \mathcal{D}\left(\mathcal{H}_{A}\right)$. Let $M_{n}^{*}(D, \varepsilon)$ denote the minimum achievable code size, for an entanglement-assisted quantum rate distortion code of blocklength $n$, at excess-distortion probability $\varepsilon$ and distortion $D$, for the average symbolwise distortion observable $\bar{\Delta}_{R^{n} B^{n}}$ defined by (72). In this section, we show that the one-shot bounds from the previous sections converge to the known expression for the entanglement-assisted quantum rate distortion function from [15]:

$$
\lim _{\varepsilon \rightarrow 0} \lim _{n \rightarrow \infty} \frac{1}{n} \log \left(M_{n}^{*}(D, \varepsilon)\right)=R_{e a}^{q}(D),
$$

where

$$
R_{e a}^{q}(D):=\frac{1}{2} \min _{\mathcal{N}_{A \rightarrow B}}\left\{I(R ; B)_{\omega}: \delta_{\text {mean }}\left(\rho, \mathcal{N}_{A \rightarrow B}, \Delta_{R B}\right) \leq D\right\}
$$

where $\delta_{\text {mean }}\left(\rho, \mathcal{N}_{A \rightarrow B}, \Delta_{R B}\right)$ denotes the mean distotion and is defined through (28). (We should clarify that [15] proved the above result for an entanglement fidelity based distortion measure, but it is clear that the results there hold for an arbitrary distortion observable.)

\subsection{First-Order Convergence of the Achievability Bound for a Memoryless Source}

We now show that the one-shot expressions from Sections 6.2 and 6.1 provide a lower bound on the entanglement-assisted quantum rate distortion function defined in (92). We do this by applying Lemma 19 and the one-shot bounds in Sections 6.2 and 6.1 .

We first analyze how the expression (59) from Section 6.2 converges; the analysis for the expression (57) from Section 6.1 then follows similarly. From (59) and the fact that we are now considering the average symbol-wise excess-distortion projector $\left(\bar{\Pi}_{>D}\right)_{R^{n} B^{n}}$, it follows that if Alice and Bob share entanglement in the form of maximally entangled states, then

$$
\frac{1}{n} \log M_{n}^{*} \leq \min _{\mathcal{N}_{A^{n} \rightarrow B^{n}}, \varepsilon_{1}}\left\{\frac{1}{2 n}\left[H_{0}^{\delta}\left(B^{n}\right)_{\Omega}-H_{\min }^{\delta}\left(B^{n} \mid R^{n}\right)_{\Omega}\right]+\frac{1}{n} \log \frac{1}{\delta^{\prime}}:(A), 0<\varepsilon_{1}<\varepsilon\right\},
$$

for every $n$ and $\varepsilon$, where $\mathcal{N}_{A^{n} \rightarrow B^{n}}$ is a CPTP map from $\mathcal{D}\left(\mathcal{H}_{A}^{\otimes n}\right) \rightarrow \mathcal{D}\left(\mathcal{H}_{B}^{\otimes n}\right), \delta, \delta^{\prime}$ are positive constants defined as in Section 6.2,

$$
\Omega_{R^{n} B^{n}}=\left(\operatorname{id}_{R^{n}} \otimes \mathcal{N}_{A^{n} \rightarrow B^{n}}\right)\left(\varphi_{R A}^{\rho}\right)^{\otimes n},
$$


and $(A)$ denotes the condition

$$
(A): \operatorname{Tr}\left(\left(\bar{\Pi}_{>D}\right)_{R^{n} B^{n}}\left(\operatorname{id}_{R^{n}} \otimes \mathcal{N}_{A^{n} \rightarrow B^{n}}\right)\left(\varphi_{R A}^{\rho}\right)^{\otimes n}\right) \leq \varepsilon_{1},
$$

for $\left(\bar{\Pi}_{>D}\right)_{R^{n} B^{n}}$ the excess-distortion projection operator defined in (77).

We can obtain an upper bound on the RHS of (93) by restricting the minimization to CPTP maps of the form $\mathcal{N}_{A^{n} \rightarrow B^{n}}:=\left(\mathcal{N}_{A \rightarrow B}\right)^{\otimes n}$. Furthermore, we can simply pick $\varepsilon_{1}=\varepsilon / 2$ so that we just require that the excess-distortion probability of the ideal simulation of the map $\left(\mathcal{N}_{A \rightarrow B}\right)^{\otimes n}$ is no larger than $\varepsilon / 2$ (recall that the ideal simulation is achieved by Alice acting on the source state with the Stinespring isometry of the map). This yields the following bound

$$
\frac{1}{n} \log M_{n}^{*} \leq \min _{\mathcal{N}_{A \rightarrow B}}\left\{\frac{1}{2 n}\left[H_{0}^{\delta}\left(B^{n}\right)_{\omega^{\otimes n}}-H_{\min }^{\delta}\left(B^{n} \mid R^{n}\right)_{\omega^{\otimes n}}\right]+\frac{1}{n} \log \frac{1}{\delta^{\prime}}:\left(A^{\prime}\right)\right\},
$$

where

$$
\left(A^{\prime}\right): \operatorname{Tr}\left(\left(\bar{\Pi}_{>D}\right)_{R^{n} B^{n}} \omega_{R B}^{\otimes n}\right) \leq \frac{\varepsilon}{2},
$$

Now consider any map $\mathcal{N}_{A \rightarrow B}$ such that $\operatorname{Tr}\left\{\Delta_{R B} \omega_{R B}\right\} \leq D-\nu$ for some $\nu>0$ where

$$
\omega_{R B}=\left(\operatorname{id}_{R} \otimes \mathcal{N}_{A \rightarrow B}\right)\left(\varphi_{R A}^{\rho}\right) .
$$

By Lemma 19, the excess-distortion probability resulting from the ideal simulation obeys

$$
\operatorname{Tr}\left(\left(\bar{\Pi}_{>D}\right)_{R^{n} B^{n}} \omega_{R B}^{\otimes n}\right) \leq \exp \left\{-2 n \nu^{2} / d_{\max }^{2}\right\} .
$$

For large enough $n$, this can be made less than $\varepsilon / 2$, so that we can further restrict the minimization to maps satisfying $\operatorname{Tr}\left\{\Delta_{R B} \omega_{R B}\right\} \leq D-\nu$. Then the following upper bound applies for large enough $n$ :

$$
\frac{1}{n} \log M_{n}^{*} \leq \min _{\mathcal{N}_{A \rightarrow B}}\left\{\frac{1}{2 n}\left[H_{0}^{\delta}\left(B^{n}\right)_{\omega \otimes n}-H_{\min }^{\delta}\left(B^{n} \mid R^{n}\right)_{\omega \otimes n}\right]+\frac{1}{n} \log \frac{1}{\delta^{\prime}}: \operatorname{Tr}\left\{\Delta_{R B} \omega_{R B}\right\} \leq D-\nu\right\},
$$

and the total excess-distortion probability of the protocol (which consists of the ideal simulation followed by quantum state-splitting) is less than $\varepsilon$. Then the relations (19) and (16) imply that the following bound holds

$$
\begin{aligned}
\lim _{\varepsilon \rightarrow 0} \lim _{n \rightarrow \infty} \frac{1}{n} \log M_{n}^{*}(D, \varepsilon) & \leq \min _{\mathcal{N}_{A \rightarrow B}}\left\{\frac{1}{2}\left[H(B)_{\omega}-H(B \mid R)_{\omega}\right]: \operatorname{Tr}\left\{\Delta_{R B} \omega_{R B}\right\} \leq D-\nu\right\} \\
& =\min _{\mathcal{N}_{A \rightarrow B}}\left\{\frac{1}{2} I(R ; B)_{\omega}: \operatorname{Tr}\left\{\Delta_{R B} \omega_{R B}\right\} \leq D-\nu\right\}
\end{aligned}
$$

By taking the limit $\nu \rightarrow 0$, we observe that the one-shot expression is bounded from above by the entanglement-assisted quantum rate distortion function given in (92).

If instead Alice and Bob share entanglement in the form of embezzling states, then it follows from (57) that

$$
\frac{1}{n} \log M_{n}^{*} \leq \min _{\mathcal{N}_{A^{n} \rightarrow B^{n}}}\left\{\frac{1}{2 n} I_{\max }^{\varepsilon / 5}\left(B^{n} ; R^{n}\right)_{\Omega}+\frac{1}{n}\left(2 \log (5 / \varepsilon)+4+\log \log \left(\left|B^{n}\right|+(5 / \varepsilon)^{2}\right)\right):(A)\right\},
$$

where $\varepsilon_{1}=\varepsilon / 5$ in $(A)$ and $\Omega_{R^{n} B^{n}}$ is the state defined by (94). A very similar argument as above then shows that this expression is bounded from above by (92) in the limit. 


\subsection{First-order convergence of the converse bound for a memoryless source}

We now show that in the limit of asymptotically many copies of a memoryless source, the converse bound given by Corollary 8 is bounded from below by the expression (92) for the entanglementassisted quantum rate distortion function.

Theorem 21 For an average symbol-wise distortion measure, the lower bound from Corollary 8 is bounded from below by $R_{e a}^{q}(D)$ defined in (92):

$$
\lim _{\varepsilon \rightarrow 0} \lim _{n \rightarrow \infty} \frac{1}{n} \min _{\mathcal{N}_{A^{n} \rightarrow B^{n}}} \min _{\psi_{B^{n}}} \frac{1}{2}\left[D_{H}^{1-\varepsilon^{\prime}}\left(\omega_{R^{n} B^{n}} \|\left(\varphi_{R}^{\rho}\right)^{\otimes n} \otimes \psi_{B^{n}}\right)-\log \frac{1}{\varepsilon^{\prime \prime}}\right] \geq R_{e a}^{q}(D) .
$$

where $\varepsilon^{\prime}>2 \varepsilon, \varepsilon^{\prime \prime}=\varepsilon^{\prime}\left(\varepsilon^{\prime} / 2-\varepsilon\right)$,

$$
\omega_{R^{n} B^{n}}:=\left(\operatorname{id}_{R^{n}} \otimes \mathcal{N}_{A^{n} \rightarrow B^{n}}\right)\left(\left(\varphi_{R A}^{\rho}\right)^{\otimes n}\right),
$$

and the outermost minimization is over quantum channels $\mathcal{N}_{A^{n} \rightarrow B^{n}}$ such that

$$
\operatorname{Tr}\left\{\left(\bar{\Pi}_{\leq D}\right)_{R^{n} B^{n}}\left(\operatorname{id}_{R^{n}} \otimes\left(\mathcal{N}_{A^{n} \rightarrow B^{n}}^{e a}\right)\right)\left(\varphi_{R A}^{\rho}\right)^{\otimes n}\right\} \geq 1-\varepsilon
$$

In proving the above theorem we make use of the following lemma, which follows directly from Lemma 14 of [15].

Lemma 22 The entanglement-assisted quantum rate distortion function $R_{e a}^{q}(D)$ is non-increasing and convex:

$$
\begin{gathered}
D_{1}<D_{2} \Longrightarrow R_{e a}^{q}\left(D_{1}\right) \geq R_{e a}^{q}\left(D_{2}\right) . \\
R_{e a}^{q}\left(\lambda D_{1}+(1-\lambda) D_{2}\right) \leq \lambda R_{e a}^{q}\left(D_{1}\right)+(1-\lambda) R_{e a}^{q}\left(D_{2}\right),
\end{gathered}
$$

where $0 \leq \lambda \leq 1$.

We also make use of the following property of the quantum mutual information which was proved in [15].

Lemma 23 (Superadditivity of quantum mutual information): The quantum mutual information is superadditive in the sense that for any CPTP $\operatorname{map} \mathcal{N}_{A_{1} A_{2} \rightarrow B_{1} B_{2}}$,

$$
I\left(R_{1} R_{2} ; B_{1} B_{2}\right)_{\sigma} \geq I\left(R_{1} ; B_{1}\right)_{\sigma}+I\left(R_{2} ; B_{2}\right)_{\sigma}
$$

where

$$
\sigma_{R_{1} R_{2} B_{1} B_{2}}=\mathcal{N}_{A_{1} A_{2} \rightarrow B_{1} B_{2}}\left(\phi_{R_{1} A_{1}} \otimes \varphi_{R_{2} A_{2}}\right) .
$$

Proof of Theorem 21. First note that the condition (103), Lemma 18 and the definition (72) of $\bar{\Delta}_{R^{n} B^{n}}$ implies that

$$
\operatorname{Tr}\left(\bar{\Delta}_{R^{n} B^{n}} \omega_{R^{n} B^{n}}\right) \equiv \frac{1}{n} \sum_{i=1}^{n} \operatorname{Tr}\left(\Delta_{R_{i} B_{i}} \omega_{R_{i} B_{i}}\right) \leq D+d_{\max } \varepsilon
$$

where $d_{\max }$ denotes the maximum eigenvalue of the distortion observable $\Delta_{R B}$. 
Let $\mathcal{N}_{A^{n} \rightarrow B^{n}}^{*}$ be the map achieving the minimum in (101) and define the output state $\omega_{R^{n} B^{n}}:=$ $\left(\operatorname{id}_{R^{n}} \otimes \mathcal{N}_{A^{n} \rightarrow B^{n}}^{*}\right)\left(\left(\varphi_{R A}^{\rho}\right)^{\otimes n}\right)$. Using Lemma 1 with $\delta=1-\varepsilon^{\prime}$, and the following relation from [13],

$$
D_{\max }^{\sqrt{2 \varepsilon^{\prime}}}(\rho \| \sigma) \geq D(\widetilde{\rho} \| \sigma),
$$

where $\widetilde{\rho} \in \mathcal{B}^{\sqrt{2 \varepsilon^{\prime}}}(\rho)$ is the state minimizing the smooth max-entropy, we find that

$$
\begin{aligned}
\text { LHS of (101) } & \geq \frac{1}{n} \min _{\psi_{B^{n}}} \frac{1}{2}\left[D_{\max }^{\sqrt{2 \varepsilon^{\prime}}}\left(\omega_{R^{n} B^{n}} \|\left(\varphi_{R}^{\rho}\right)^{\otimes n} \otimes \psi_{B^{n}}\right)+\log \left(\frac{\varepsilon^{\prime}}{2}-\varepsilon\right)\right] \\
& \geq \frac{1}{n} \min _{\psi_{B^{n}}} \frac{1}{2}\left[D\left(\widetilde{\omega}_{R^{n} B^{n}} \|\left(\varphi_{R}^{\rho}\right)^{\otimes n} \otimes \psi_{B^{n}}\right)+\log \left(\frac{\varepsilon^{\prime}}{2}-\varepsilon\right)\right] \\
& \geq \frac{1}{n} \min _{\psi_{B^{n}}, \tau_{R^{n}}} \frac{1}{2}\left[D\left(\widetilde{\omega}_{R^{n} B^{n}} \| \tau_{R^{n}} \otimes \psi_{B^{n}}\right)+\log \left(\frac{\varepsilon^{\prime}}{2}-\varepsilon\right)\right] \\
& \geq \frac{1}{2 n}\left[D\left(\widetilde{\omega}_{R^{n} B^{n}} \| \widetilde{\omega}_{R^{n}} \otimes \widetilde{\omega}_{B^{n}}\right)+\log \left(\frac{\varepsilon^{\prime}}{2}-\varepsilon\right)\right] \\
& =\frac{1}{2 n}\left[I\left(R^{n} ; B^{n}\right)_{\widetilde{\omega}_{R^{n} B^{n}}}+\log \left(\frac{\varepsilon^{\prime}}{2}-\varepsilon\right)\right] \\
& \geq \frac{1}{2 n} I\left(R^{n} ; B^{n}\right)_{\omega_{R^{n} B^{n}}}-f\left(\varepsilon, \varepsilon^{\prime}, n\right),
\end{aligned}
$$

where

$$
f\left(\varepsilon, \varepsilon^{\prime}, n\right):=\frac{1}{2 n}\left[5 \sqrt{2 \varepsilon^{\prime}} n \log |R|-3 h_{2}\left(\sqrt{2 \varepsilon^{\prime}}\right)+\log \left(\frac{\varepsilon^{\prime}}{2}-\varepsilon\right)\right] .
$$

The third inequality follows by introducing a further minimization. The fourth inequality follows from

$$
\min _{\sigma_{R}, \tau_{B}} D\left(\rho_{R B} \| \sigma_{R} \otimes \tau_{B}\right)=D\left(\rho_{R B} \| \rho_{R} \otimes \rho_{B}\right)
$$

The last inequality follows by applying the Alicki-Fannes' inequality (continuity of conditional entropy) 2]. Continuing we have,

$$
\begin{aligned}
\text { LHS of (106) } & \geq \frac{1}{2 n} \sum_{i=1}^{n} I\left(R_{i} ; B_{i}\right)-f\left(\varepsilon, \varepsilon^{\prime}, n\right) \\
& \geq \frac{1}{n} \sum_{i=1}^{n} R_{e a}^{q}\left(\operatorname{Tr}\left(\Delta_{R_{i} B_{i}} \omega_{R_{i} B_{i}}\right)\right)-f\left(\varepsilon, \varepsilon^{\prime}, n\right) \\
& \geq R_{e a}^{q}\left(\frac{1}{n} \sum_{i=1}^{n} \operatorname{Tr}\left(\Delta_{R_{i} B_{i}} \omega_{R_{i} B_{i}}\right)\right)-f\left(\varepsilon, \varepsilon^{\prime}, n\right) \\
& \geq R_{e a}^{q}\left(D+d_{\max } \varepsilon\right)-f\left(\varepsilon, \varepsilon^{\prime}, n\right) .
\end{aligned}
$$

The first inequality follows from superadditivity of quantum mutual information (Lemma 23). The second inequality follows from the fact that the state $\omega_{R_{i} B_{i}}$ has mean distortion equal to $\operatorname{Tr}\left(\Delta_{R_{i} B_{i}} \omega_{R_{i} B_{i}}\right)$, and $R_{e a}^{q}\left(\operatorname{Tr}\left(\Delta_{R_{i} B_{i}} \omega_{R_{i} B_{i}}\right)\right)$ is the minimum of half the quantum mutual information over all CPTP maps on the system $R_{i} A_{i}$ with this distortion. The last two inequalities follow from the convexity of the function $R_{e a}^{q}(D)$ (Lemma 22), the inequality (104), and from the fact that $R_{e a}^{q}(D)$ is a non-increasing function of $D$ (Lemma 22).

Finally, we can take $\varepsilon^{\prime}=3 \varepsilon$. Then in the limit as $n \rightarrow \infty$ and $\varepsilon \rightarrow 0$, the lower bound in the last line of (107) converges to $R_{e a}^{q}(D)$. 


\section{Finite Blocklength Results for the Isotropic Qubit Source}

In this section, we obtain tight lower and upper bounds on the minimum achievable code size for the case of an isotropic qubit source with entanglement assistance [18, 40. These bounds hold for any finite blocklength $n$, the entanglement fidelity based distortion observable $\bar{\Delta}_{R^{n} B^{n}}$ from (89), any excess-distortion probability $\varepsilon$, and any distortion $D$ where $0 \leq D \leq 1$. In this case, the source is equal to $\pi_{A}^{\otimes n}$, where $\pi_{A}:=I_{A} / 2$. A purification of one copy of the source is the Bell state

$$
|\Phi\rangle_{R A}:=\frac{1}{\sqrt{2}}\left(|00\rangle_{R A}+|11\rangle_{R A}\right) .
$$

Ref. [40] proved that the entanglement-assisted quantum rate distortion function in (92) for this example is equal to

$$
R_{e a}^{q}(D)= \begin{cases}1-\frac{1}{2} H\left(\left\{1-D, \frac{D}{3}, \frac{D}{3}, \frac{D}{3}\right\}\right) & \text { if } 0 \leq D \leq \frac{3}{4}, \\ 0 & \text { if } \frac{3}{4} \leq D \leq 1,\end{cases}
$$

where we have used the notation $H(\{\cdot\})$ to denote the Shannon entropy of the probability distribution inside the braces $\{\cdot\}$.

The methods in the following subsections combined with the results of Kostina and Verdú [25] allow us to conclude the following finite blocklength characterization for entanglement-assisted quantum rate distortion coding of an isotropic qubit source:

$$
R(n, D, \varepsilon):=\frac{1}{n} \log \left(M_{n}^{*}(D, \varepsilon)\right)=1-\frac{1}{2}\left[h_{2}(D)-D \log 3\right]+\frac{1}{4 n} \log (n)+O\left(\frac{1}{n}\right) .
$$

if $0<D<\frac{3}{4}$.

\subsection{Finite Blocklength Converse for the Isotropic Qubit Source}

Applying Proposition 9 (specifically, the bound in (42)) to the scenario mentioned above, we have the following lower bound on the minimum achievable code size $M$ :

$$
\begin{aligned}
M & \geq \max _{\sigma_{R^{n} A^{n}}} \min _{\psi_{B^{n}}} \sqrt{\frac{\beta_{\varepsilon}\left(\Phi_{R A}^{\otimes n} \| \sigma_{R^{n} A^{n}}\right)}{\operatorname{Tr}\left\{\left(\bar{\Pi}_{\leq D}\right)_{R^{n} B^{n}}\left(\sigma_{R^{n}} \otimes \psi_{B^{n}}\right)\right\}}} \\
& \geq \min _{\psi_{B^{n}}} \sqrt{\frac{\beta_{\varepsilon}\left(\Phi_{R A}^{\otimes n} \| \Phi_{R A}^{\otimes n}\right)}{\operatorname{Tr}\left\{\left(\bar{\Pi}_{\leq D}\right)_{R^{n} B^{n}}\left(\pi_{R}^{\otimes n} \otimes \psi_{B^{n}}\right)\right\}}} \\
& \geq \sqrt{\frac{1-\varepsilon}{2^{-n} \max _{\psi_{B^{n}}} \operatorname{Tr}\left\{\left(\bar{\Pi}_{\leq D}\right)_{R^{n} B^{n}}\left(I_{R}^{\otimes n} \otimes \psi_{B^{n}}\right)\right\}}} .
\end{aligned}
$$

The second inequality follows by choosing $\sigma_{R^{n}} A^{n}$ from the optimization to be equal to $\Phi_{R A}^{\otimes n}$. The third inequality follows from the definition of $\beta_{\varepsilon}\left(\Phi_{R A}^{\otimes n} \| \Phi_{R A}^{\otimes n}\right)$ in (20) and by realizing that $\pi_{R}^{\otimes n}=$ $2^{-n} I_{R}^{\otimes n}$. Since the expression in the trace features the operator $I_{R}^{\otimes n}$ on the right side, we can evaluate it effectively by taking a partial trace of the excess distortion observable with respect to the $R^{n}$ systems. By exploiting the expansion in (90) and the fact that

$$
\begin{aligned}
& \operatorname{Tr}_{R}\left\{\Pi_{0}\right\}=\operatorname{Tr}_{R}\left\{\Phi_{R B}\right\}=\frac{1}{2} I_{B} \\
& \operatorname{Tr}_{R}\left\{\Pi_{1}\right\}=\operatorname{Tr}_{R}\left\{I_{R B}-\Phi_{R B}\right\}=\frac{3}{2} I_{B},
\end{aligned}
$$


we find that

$$
\begin{aligned}
& \operatorname{Tr}_{R^{n}}\left\{\left(\bar{\Pi}_{\leq D}\right)_{R^{n} B^{n}}\right\}=\operatorname{Tr}_{R^{n}}\left\{\sum_{j \in\{1, \ldots, n\}: j / n \leq D}\left[\sum_{x^{n} \in\{0,1\}^{n}: \operatorname{wt}\left(x^{n}\right)=j} \Pi_{x^{n}}\right]\right\} \\
& =\sum_{j \in\{1, \ldots, n\}: j / n \leq D}\left[\sum_{x^{n} \in\{0,1\}^{n}: \operatorname{wt}\left(x^{n}\right)=j} \operatorname{Tr}_{R^{n}}\left\{\Pi_{x^{n}}\right\}\right] \\
& =\sum_{j \in\{1, \ldots, n\}: j / n \leq D}\left[\sum_{x^{n} \in\{0,1\}^{n}: \operatorname{wt}\left(x^{n}\right)=j}\left(\frac{1}{2}\right)^{n-j}\left(\frac{3}{2}\right)^{j} I_{B}^{\otimes n}\right] \\
& =\sum_{j \in\{1, \ldots, n\}: j / n \leq D}\left(\begin{array}{c}
n \\
j
\end{array}\right)\left(\frac{1}{2}\right)^{n-j}\left(\frac{3}{2}\right)^{j} I_{B}^{\otimes n} \\
& =\frac{1}{2^{n}} \sum_{j \in\{1, \ldots, n\}: j / n \leq D}\left(\begin{array}{c}
n \\
j
\end{array}\right) 3^{j} I_{B}^{\otimes n} \\
& =\frac{1}{2^{n}} S_{\lfloor n D\rfloor} I_{B}^{\otimes n}
\end{aligned}
$$

where

$$
S_{k}:=\sum_{j=0}^{k}\left(\begin{array}{l}
n \\
j
\end{array}\right) 3^{j} .
$$

Substituting into (110), this leaves us with

$$
\sqrt{\frac{1-\varepsilon}{2^{-2 n} S_{\lfloor n D\rfloor} \max _{\psi_{B^{n}}} \operatorname{Tr}\left\{\psi_{B^{n}}\right\}}}=\sqrt{\frac{1-\varepsilon}{2^{-2 n} S_{\lfloor n D\rfloor}}} .
$$

Taking logarithms of both sides and dividing by $n$, we get

$$
\frac{1}{n} \log M \geq 1-\frac{1}{2 n} \log S_{\lfloor n D\rfloor}+\frac{1}{2 n} \log (1-\varepsilon) .
$$

Applying the following estimate stated as Eq. (390) in Appendix $\mathrm{H}$ of [25], which holds for $0<$ $D<3 / 4$

$$
\log S_{\lfloor n D\rfloor}=n h_{2}(D)+n D \log 3-\frac{1}{2} \log n+O(1)
$$

we find that

$$
R=\frac{1}{n} \log M \geq 1-\frac{1}{2}\left[h_{2}(D)+D \log 3\right]+\frac{\log (n)}{4 n}+O\left(\frac{1}{n}\right) .
$$

Considering that the bound from [15] for the entanglement-assisted quantum rate distortion function was the first-order term $1-\frac{1}{2}\left[h_{2}(D)+D \log 3\right]$, the above bound provides a strong refinement of it that includes logarithmic corrections for finite blocklength.

The following bound applies to entanglement-assisted rate distortion with classical communication by applying super-dense coding [6]:

$$
\frac{1}{n} \log M_{C} \geq 2-\left[h_{2}(D)+D \log 3\right]+\frac{\log (n)}{2 n}+O\left(\frac{1}{n}\right) .
$$




\subsection{Finite Blocklength Achievability Part for the Isotropic Qubit Source}

\subsubsection{The Teleportation Method}

For the case of an isotropic qubit source, there is a simple teleportation strategy [4] for achieving its entanglement-assisted quantum rate distortion function. First, we consider a strategy that employs entanglement assistance with noiseless classical communication, and we count the number of classical bits sent. Then, we relate this strategy to one with entanglement assistance and noiseless quantum communication by employing super-dense coding [6].

The protocol outlined here is related to the forward classical communication cost of simulating a Bell-diagonal channel via teleportation [5]. However, the task that is accomplished here is ratedistortion coding rather than channel simulation (see Remark 10).

The protocol operates as follows:

1. Alice shares $n$ copies of the Bell state $|\Phi\rangle_{R A}$ with the reference. She also shares $n$ copies of the maximally entangled state $|\Phi\rangle_{A^{\prime} B}$ with Bob (recall that in the entanglement-assisted setting, they are allowed as much entanglement as they need in any form that they wish).

2. Alice and Bob operate as in the teleportation protocol [4]. She performs a Bell measurement on each of the $A A^{\prime}$ systems, obtaining a classical sequence $x^{n}:=x_{1} \cdots x_{n}$, where $x_{i} \in\{0,1,2,3\}$.

3. If Alice were to send the sequence $x^{n}$ itself, then Bob would be able to reconstruct the states $\left(|\Phi\rangle_{R B}\right)^{\otimes n}$ perfectly. Instead, Alice and Bob employ a classical 4-ary rate distortion code with codewords $\left\{y^{n}(m)\right\}_{m \in[M]}$. So, Alice finds the codeword representative $y^{n}(m)$ with minimum distortion from the measurement outcomes $x^{n}$, as measured by the Hamming distance distortion measure:

$$
\bar{d}\left(x^{n}, y^{n}\right):=\frac{1}{n} \sum_{i=1}^{n} I\left\{x_{i} \neq y_{i}\right\}
$$

where $I\{\cdot\}$ is an indicator function, equal to one if its argument is true and equal to zero otherwise. Alice then sends the index $m$ of the codeword representative $y^{n}(m)$ over the noiseless classical channels.

4. Bob, knowing the code $\left\{y^{n}(m)\right\}_{m \in[M]}$, performs the correction operations according to the sequence $y^{n}(m)$ as given in the teleportation protocol. The result is that he creates a state of the following form:

$$
\left|\Phi_{x^{n}, y^{n}}\right\rangle:=\bigotimes_{i=1}^{n} \sigma_{y_{i}} \sigma_{x_{i}}|\Phi\rangle_{R_{i} B_{i}},
$$

where $\sigma_{y_{i}}$ and $\sigma_{x_{i}}$ are one of the four Pauli operators $\left\{I, \sigma_{X}, \sigma_{Y}, \sigma_{Z}\right\}$.

5. The distortion as measured by the symbol-wise entanglement fidelity is then equivalent to the distortion $\bar{d}\left(x^{n}, y^{n}\right)$ as given above, because

$$
\operatorname{Tr}\left\{\frac{1}{n} \sum_{i=1}^{n}\left(I_{R_{i} B_{i}}-|\Phi\rangle\left\langle\left.\Phi\right|_{R_{i} B_{i}}\right)\left|\Phi_{x^{n}, y^{n}}\right\rangle\left\langle\Phi_{x^{n}, y^{n}}\right|\right\}=\frac{1}{n} \sum_{i=1}^{n} I\left\{x_{i} \neq y_{i}\right\} .\right.
$$


Thus, the performance of this protocol as measured by the excess-distortion probability is exactly the same as the performance of a classical rate distortion code for a uniform 4-ary source. Kostina and Verdú have calculated tight finite blocklength bounds for this case [25], and as such, we can consider them directly for our purposes here. In particular, they have shown that there exists a classical $(n, M, D, \varepsilon)$ code satisfying

$$
\varepsilon \leq\left(1-S_{\lfloor n D\rfloor} 4^{-n}\right)^{M}
$$

where $S_{k}$ is defined in (111). Applying their bound stated as Eq. (395) of Appendix H of [25] and the same estimate as in (112), we find the following bound:

$$
2-h_{2}(D)-D \log 3+\frac{1}{2 n} \log n+O\left(\frac{1}{n}\right) \geq \frac{1}{n} \log M .
$$

Using the fact that this then leads to a protocol for entanglement-assisted quantum rate distortion coding by super-dense coding, we obtain the following bound for such a code:

$$
1-\frac{1}{2}\left[h_{2}(D)-D \log 3\right]+\frac{1}{4 n} \log n+O\left(\frac{1}{n}\right) \geq \frac{1}{n} \log M_{Q}
$$

\section{Conclusion}

We have provided a framework for one-shot quantum rate distortion coding, by introducing the notion of an excess-distortion projector corresponding to a distortion observable. We then proved lower and upper bounds on the minimum qubit compression size of an entanglement-assisted quantum rate distortion code. The lower bounds also serve as lower bounds for unassisted codes, since entanglement can only help to reduce the minimum qubit compression size. These bounds were expressed in terms of entropic quantities familiar from the smooth entropy formalism [29, 33, 19, 14]. Next, we showed how these entanglement-assisted bounds converge to the known expression for the entanglement-assisted quantum rate distortion function of a memoryless quantum information source. Finally, we determined a tight, finite blocklength characterization for the entanglementassisted minimum qubit compression size of an isotropic qubit source. The quantum teleportation strategy used in the achievability part of this characterization is the first strategy, to our knowledge, different from channel simulation to be employed for the purpose of quantum rate distortion coding.

There are many questions to consider going forward from here. First, it would be ideal to find better characterizations of the minimum qubit compression size for an unassisted source (this is of course related to the fact that we would like a better characterization of the unassisted quantum rate distortion function other than the one given in [15, which is in terms of the entanglement of purification). Second, understanding a quantum analog of the "tilted information" from [25] might be helpful since this quantity gives a second-order refinement of the classical rate-distortion function. Finally, it would also be good to generalize quantum rate distortion theory to the continuousvariable setting since this is one of the main motivations for pursuing quantum rate distortion. Some results were offered in [9], but unfortunately they only considered Barnum's coherent-information lower bound, which we know is not a good bound since it can become negative.

Acknowledgements. We are grateful to Victoria Kostina and Sergio Verdú for several helpful conversations during the "Beyond i.i.d. in information theory" workshop at the University of 
Cambridge and to Will Matthews as well for interesting and helpful discussions. ND is grateful to Pembroke College for sponsoring the workshop in which the idea for this project had its genesis. JMR and RR were supported by the Swiss National Science Foundation (through the National Centre of Competence in Research "Quantum Science and Technology" and grant No. 200020-135048) and by the European Research Council (grant 258932). MMW acknowledges support from the Centre de Recherches Mathématiques and is grateful for the hospitality of the Statistical Laboratory at the University of Cambridge and the Pauli Center for Theoretical Studies (ETH Zurich) during a research visit in January and February of 2013, when the majority of this work was completed.

\section{References}

[1] Anura Abeyesinghe, Igor Devetak, Patrick Hayden, and Andreas Winter. The mother of all protocols: Restructuring quantum information's family tree. Proceedings of the Royal Society A, 465(2108):2537-2563, August 2009. arXiv:quant-ph/0606225.

[2] Robert Alicki and Mark Fannes. Continuity of quantum conditional information. Journal of Physics A: Mathematical and General, 37(5):L55-L57, 2004.

[3] Howard Barnum. Quantum rate-distortion coding. Physical Review A, 62(4):042309, September 2000. arXiv:quant-ph/9806065.

[4] Charles H. Bennett, Gilles Brassard, Claude Crépeau, Richard Jozsa, Asher Peres, and William K. Wootters. Teleporting an unknown quantum state via dual classical and EinsteinPodolsky-Rosen channels. Physical Review Letters, 70(13):1895-1899, March 1993.

[5] Charles H. Bennett, Peter W. Shor, John A. Smolin, and Ashish V. Thapliyal. Entanglementassisted classical capacity of noisy quantum channels. Physical Review Letters, 83(15):30813084, October 1999.

[6] Charles H. Bennett and Stephen J. Wiesner. Communication via one- and two-particle operators on Einstein-Podolsky-Rosen states. Physical Review Letters, 69(20):2881-2884, November 1992.

[7] Mario Berta, Matthias Christandl, and Renato Renner. The quantum reverse Shannon theorem based on one-shot information theory. Communications in Mathematical Physics, 306:579, September 2011. arXiv:0912.3805.

[8] Mario Berta, Joseph M. Renes, and Mark M. Wilde. Identifying the information gain of a quantum measurement. arXiv:1301.1594, January 2013.

[9] Xiao-Yu Chen and Wei-Ming Wang. Entanglement information rate distortion of a quantum Gaussian source. IEEE Transactions on Information Theory, 54(2):743-748, February 2008.

[10] Nikola Ciganović. Smooth max-mutual information as a generalization of von Neumann mutual information for the one-shot setting. Master's thesis, ETH Zurich, August 2012.

[11] Nikola Ciganović, Normand J. Beaudry, and Renato Renner. Smooth max-information as one-shot generalization for mutual information. August 2013. arXiv:1308.5884. 
[12] The contributors to Wikipedia. Hoeffding's inequality. Wikipedia. Retrieved on February 28, 2013.

[13] Nilanjana Datta. Min- and max- relative entropies and a new entanglement monotone. IEEE Transactions on Information Theory, 55:2816, 2009. arXiv:0803.2770.

[14] Nilanjana Datta and Min-Hsiu Hsieh. The apex of the family tree of protocols: Optimal rates and resource inequalities. New Journal of Physics, 13:093042, 2011. arXiv:1103.1135.

[15] Nilanjana Datta, Min-Hsiu Hsieh, and Mark M. Wilde. Quantum rate distortion, reverse Shannon theorems, and source-channel separation. IEEE Transactions on Information Theory, 59:615-630, January 2013. arXiv:1108.4940.

[16] Nilanjana Datta, Min-Hsiu Hsieh, Mark M. Wilde, and Andreas Winter. Quantum-toclassical rate distortion coding. Journal of Mathematical Physics, 54(4):042201, April 2013. arXiv:1210.6962.

[17] Igor Devetak. Triangle of dualities between quantum communication protocols. Physical Review Letters, 97(14):140503, October 2006.

[18] Igor Devetak and Toby Berger. Quantum rate-distortion theory for memoryless sources. IEEE Transactions on Information Theory, 48(6):1580-1589, June 2002. arXiv:quant-ph/0011085.

[19] Frederic Dupuis, Lea Kraemer, Philippe Faist, Joseph M. Renes, and Renato Renner. Generalized entropies. November 2012. arXiv:1211.3141.

[20] Christopher A. Fuchs and Jeroen van de Graaf. Cryptographic distinguishability measures for quantum mechanical states. IEEE Transactions on Information Theory, 45(4):1216-1227, May 1999. arXiv:quant-ph/9712042.

[21] Fabian Furrer, Johan Aberg, and Renato Renner. Min- and max-entropy in infinite dimensions. Communications in Mathematical Physics, 306(1):165-186, 2011. arXiv:1004.1386.

[22] Robert G. Gallager. Information Theory and Reliable Communication. John Wiley and Sons, Inc., 1968.

[23] Wassily Hoeffding. Probability inequalities for sums of bounded random variables. Journal of the American Statistical Association, 58(301):13-30, March 1963.

[24] Richard Jozsa. Fidelity for mixed quantum states. Journal of Modern Optics, 41(12):23152323, 1994.

[25] Victoria Kostina and Sergio Verdú. Fixed-length lossy compression in the finite blocklength regime. IEEE Transactions on Information Theory, 58(6):3309-3338, June 2012. arXiv:1102.3944.

[26] Zhicheng Luo and Igor Devetak. Channel simulation with quantum side information. IEEE Transactions on Information Theory, 55(3):1331-1342, March 2009. arXiv:quant-ph/0611008.

[27] Katalin Marton. Error exponent for source coding fidelity criterion. IEEE Transactions on Information Theory, IT-20(2):197-199, March 1974. 
[28] Tomohiro Ogawa and Hiroshi Nagaoka. Making good codes for classical-quantum channel coding via quantum hypothesis testing. IEEE Transactions on Information Theory, 53(6):22612266, June 2007.

[29] Renato Renner. Security of Quantum Key Distribution. PhD thesis, ETH Zurich, September 2005. arXiv:quant-ph/0512258.

[30] Benjamin Schumacher. Quantum coding. Physical Review A, 51(4):2738-2747, April 1995.

[31] Claude E. Shannon. Coding theorems for a discrete source with a fidelity criterion. IRE International Convention Records, 7:142-163, 1959.

[32] Yossef Steinberg and Sergio Verdú. Simulation of random processes and rate-distortion theory. IEEE Transactions on Information Theory, 42(1):63-86, January 1996.

[33] Marco Tomamichel. A Framework for Non-Asymptotic Quantum Information Theory. PhD thesis, ETH Zurich, March 2012. arXiv:1203.2142.

[34] Marco Tomamichel, Roger Colbeck, and Renato Renner. A fully quantum asymptotic equipartition property. IEEE Transactions on Information Theory, 55(12):5840-5847, December 2009. arXiv:0811.1221.

[35] Marco Tomamichel, Roger Colbeck, and Renato Renner. Duality between smooth minand max-entropies. IEEE Transactions on Information Theory, 56(9):4674-4681, 2010. arXiv:0907.5238.

[36] Armin Uhlmann. The "transition probability" in the state space of a *algebra. Reports on Mathematical Physics, 9(2):273-279, 1976.

[37] Wim van Dam and Patrick Hayden. Universal entanglement transformations without communication. Physical Review A, 67:060302, June 2003. arXiv:quant-ph/0201041.

[38] Ligong Wang and Renato Renner. One-shot classical-quantum capacity and hypothesis testing. Physical Review Letters, 108:200501, May 2012. arXiv:1007.5456.

[39] Christian Weedbrook, Stefano Pirandola, Raul Garcia-Patron, Nicolas J. Cerf, Timothy C. Ralph, Jeffrey H. Shapiro, and Seth Lloyd. Gaussian quantum information. Reviews of Modern Physics, 84:621-669, May 2012. arXiv:1110.3234.

[40] Mark M. Wilde, Nilanjana Datta, Min-Hsiu Hsieh, and Andreas Winter. Quantum rate distortion coding with auxiliary resources. IEEE Transactions on Information Theory, 59(10):67556773, October 2013. arXiv:1212.5316.

[41] Andreas Winter. Coding theorem and strong converse for quantum channels. IEEE Transactions on Information Theory, 45(7):2481-2485, 1999.

[42] Andreas Winter. Coding Theorems of Quantum Information Theory. PhD thesis, Universität Bielefeld, July 1999. arXiv:quant-ph/9907077.

[43] Andreas Winter. Compression of sources of probability distributions and density operators. August 2002. arXiv:quant-ph/0208131. 
[44] Andreas Winter and Rudolph Ahlswede. Quantum rate-distortion theory. Unpublished manuscript, June 2001. 